\title{
2-Oxiranyl-pyridines: Synthesis and Regioselective Epoxide Ring Openings with Chiral Amines as a Route to Chiral Ligands
}

\author{
Marzena Wosińska-Hrydczuk and Jacek Skarżewski iD \\ Department of Organic Chemistry, Faculty of Chemistry, Wrocław University of Technology, Wyb Wyspiańskiego 27, \\ 50-370 Wroctaw, Poland \\ Correspondence should be addressed to Jacek Skarżewski; jacek.skarzewski@pwr.edu.pl
}

Received 15 June 2019; Revised 31 July 2019; Accepted 12 September 2019; Published 9 October 2019

Academic Editor: Guillaume Berionni

Copyright (c) 2019 Marzena Wosińska-Hrydczuk and Jacek Skarżewski. This is an open access article distributed under the Creative Commons Attribution License, which permits unrestricted use, distribution, and reproduction in any medium, provided the original work is properly cited.

\begin{abstract}
New epoxides, derivatives of pyridine, 2,2'-bipyridine, and 1,10-phenanthroline, were synthesized from the respective $\alpha$-methylazaarenes. The obtained racemic 2-oxiranyl-azaarenes along with styrene oxide and trans-stilbene oxide were submitted to the ring opening with chiral primary amines as a chiral auxiliary. The most effective reaction was run in the presence of $\mathrm{Sc}(\mathrm{OTf})_{3}$ /diisopropylethylamine for 7 days at $80^{\circ} \mathrm{C}$, affording a good yield of the amino alcohols. Except for styrene oxide which gave both $\alpha$-and $\beta$-amino alcohols, the reactions led regioselectively to the corresponding diastereomeric $\beta$-amino alcohols. The resulting diastereomers were separated, and the configurations of their stereogenic centers were established. The obtained enantiomerically pure 2-pyridinyl- and 6-(2,2'-bipyridinyl)- $\beta$-amino alcohols were tentatively tested as chiral ligands in the zinccatalyzed aldol reaction.
\end{abstract}

\section{Introduction}

Modular chiral ligands and catalysts attained in a few steps from the well-defined building blocks are considered as a useful tool for asymmetric synthesis [1-4]. Among functional blocks for the modular catalysts, the moieties of pyridine, 2,2'-bipyridine, and 1,10-phenanthroline, forming strong transition metal complexes, belong to the particularly promising ones [5-12]. For this aim, we intended to develop 2-oxiranyl-pyridines that, after epoxide ring openings, would give the desired chiral products.

For this purpose, we synthesize the 2-oxiranyl-azaarenes. Their epoxide ring-opening reactions with chiral amines should lead to the separable diastereomeric $\beta$-amino alcohols with the 2-pyridinyl-type substituents. Hence, obtained homochiral complexing amino alcohols would be tested as chiral ligands.

The key synthetic reaction will be carried out using the optimized regioselective epoxide ring opening with chiral primary amines as a chiral auxiliary. This approach has a literature precedent in the synthesis of individual enantiomers of 2-amino cyclohexanol derivatives using chiral 1-phenylethylamine at the epoxide ring-opening step followed by chromatographic separation of the diastereomeric alcohols [13]. Moreover, the method applied to our 2-oxiranyl-pyridines may offer a simple route to the chiral building blocks for important medicinal compounds $[14,15]$.

Generally, plentiful successful Lewis acid activators for the epoxide ring opening with amines have been developed [16-38]. The regiochemistry of the metal salt-catalyzed aminolysis of styrene oxide depends on the amine nucleophilicity and steric bulkiness as well as the strength of Lewis acid activator (metal ion) $[39,40]$. Moreover, an interaction of the metal-ion additives with other complexing functionalities connected to the epoxide often influenced the observed regioselectivity [41-46]. Correspondingly, the metal-complexing pyridine-2-yl substituent demonstrated the regio-steering effect in the epoxide ring-opening reaction in the presence of $\mathrm{MgBr}_{2}$ [9]. Although the asymmetric aminolysis of meso-epoxides was successful in the presence of chiral metal complexes [24-32], the respective racemic 
trans-substituted epoxides could hardly be opened stereoselectively with other but aniline-type amine $[23,26]$.

\section{Results and Discussion}

2.1. Synthesis of 2-Oxiranyl-pyridines. In order to obtain the required $\alpha$-azaarene epoxides (Scheme 1), we methylated the parent azaarenes, namely, 2,2' -bipyridine (bpy) and 1,10phenanthroline (phen) with MeLi followed by the oxidative rearomatization [47, 48]. The $\alpha$-methyl derivatives 1 (commercial), 2 [47], and 3 [48] were reacted with 1 equiv of benzaldehyde in the presence of a substoichiometric amount of calcium triflate [49]. The products, trans-styryl compounds 4 [50, 51], 5 and 6, were formed in rather moderate yields. However, the unreacted methyl derivatives could be easily recovered. Moreover, when cyclohexyl carbaldehyde was used in the reaction with 3 , along with $\mathbf{6 b}$ the diene 7 was obtained. Thus, in the next step, 4, 5, and $\mathbf{6}$ were reacted with NBS in dioxane/water acidified with acetic acid giving the respective bromohydrins $\mathbf{8}, \mathbf{9}$, and $\mathbf{1 0}$ that were smoothly converted into the epoxides 11 [52, 53, 54], 12, and 13 (Scheme 1). Also, rac-2-(oxiranyl)pyridine (14) [55] was prepared analogously from 2 -vinylpyridine.

2.2. Selective Epoxide Ring Opening: Model Studies. In order to find the proper conditions for our key reaction, the promising literature method for the $\mathrm{Sc}(\mathrm{OTf})_{3}$-catalyzed epoxide ring opening $[32,39,40]$ was examined. We run the model reaction of racemic epoxides, namely, styrene oxide (15) and trans-stilbene oxide (16) with chiral 1-phenylethylamine in the presence of $\mathrm{Sc}(\mathrm{OTf})_{3} /$ diisopropylethylamine (DIEA) at $80^{\circ} \mathrm{C}$ (Scheme 2$)$.

The reaction of styrene oxide (15) with $(S)$-1-phenylethylamine gave both known regioisomers, $\beta$-amino alcohol 17 [56-58] and $\alpha$-amino alcohol 18 [59], as a separable mixture (ca. $1: 1$ ), and their structures were confirmed by ${ }^{1} \mathrm{H}$ NMR spectroscopy [56-59]. Then, pure like-17 and unlike17 diastereomers were separated by recrystallization $\left(\mathrm{CH}_{2} \mathrm{Cl}_{2}\right.$ /hexane). We ascribed their configurations by comparing the recorded ${ }^{1} \mathrm{H}$ NMR spectral properties and specific rotations with the reported ones $[56,57,60]$. The configuration of the like-isomer $\left(R, 1^{\prime} R-17\right)$ was proved undoubtedly by the X-ray structure [56]. Furthermore, the samples of pure diastereomers $\mathbf{1 7}$ and $\mathbf{1 9}$ were later used for comparison in the stereochemical assignments of the chiral azaaromatic analogs $\mathbf{2 0}$ and $\mathbf{2 1}$.

In all cases, the catalyzed reactions were completed within 7 days at $80^{\circ} \mathrm{C}$, affording a good yield of the amino alcohols. The reaction mixtures were stirred under argon in a sealed test tube, and the applied reaction time was necessary to reach the maximum conversion (controlled by TLC). The uncatalyzed reaction of $\mathbf{1 5}$ with 1-phenylethylamine gave both regioisomers $\mathbf{1 7}$ and $\mathbf{1 8}$ in $4 \%$ yield only. Moreover, the $\mathrm{Sc}(\mathrm{OTf})_{3}$-catalyzed ring opening in the absence of DIEA resulted in much poorer yield. Interestingly, when we run the reaction of $\mathbf{1 5}$ in the presence of $\mathrm{Zn}(\mathrm{OAc})_{2}$ (weaker Lewis acid), $\beta$-amino alcohol 17 was formed regioselectively. The reaction of rac-trans-stilbene oxide (16) with (S)-1- phenylethylamine gave also separable diastereomers (ca. 1 : 1) of amino alcohol $19[57,58,61]$ in $54 \%$ total yield (Scheme 2).

2.3. Selective Epoxide Ring Opening: Pyridine Derivatives. After the model studies, the epoxides with pyridine-type fragments were submitted to the ring-opening reactions. Unlike for styrene oxide (15), the reaction of rac-2-(oxiranyl)pyridine (14) with chiral amines gave only one regioisomer, $\beta$-amino alcohol 20, regardless of the catalyst used. However, the better yield was observed for $\mathrm{Sc}(\mathrm{OTf})_{3} /$ DIEA (Scheme 3).

The product 20 consisted of two diastereomers (in $c a$. $1: 1$ ratio), which were smoothly separated by column chromatography. We ascribed their configuration comparing ${ }^{1} \mathrm{H}$ NMR spectra between the isolated diastereomers 20 and 17 (see supporting file S1), where similarities between their spectral patterns could be clearly seen.

Other synthesized 2,3-disubstituted trans epoxides with the $\alpha$-azaaromatic fragments (11-13) were submitted to the ring-opening reactions, and the results are summarized in Scheme 4 and Table 1.

The reaction of $\mathbf{1 1}$ and $\mathbf{1 2}$ with chiral 1-phenylethylamine carried out in the presence of $\mathrm{Sc}(\mathrm{OTf})_{3} / \mathrm{DIEA}$ gave in each case only one regioisomer of the respective $\beta$-amino alcohol in good yield. The obtained products 21 and 23 consisted of two diastereomers (ca. 1:1), which were separated by column chromatography. Similarly, the reaction of 11 and 12 with $(R)$-1-cyclohexylethylamine gave regioselectively both amino alcohols 22 and 24 . For 22, the diastereoisomers (obtained in nearly $1: 1$ ratio) were separated to give enantiomerically pure compounds (Table 1). For the obtained diastereomeric mixture of 24, only $\left(1 R, 2 R, 1^{\prime} R\right)-\mathbf{2 4}$ could be isolated as a stereochemically pure sample. The reaction of $\mathbf{1 3 b}$ with $(R)$-1-phenylethylamine was sluggish, and the respective diastereomeric mixture 25 was formed in $8 \%$ yield. The reaction of ractrans-2-(3-phenyloxiranyl)-1,10-phenanthroline (13a) with the same amine gave inseparable mixture, and the corresponding dehydration product could be detected only by ${ }^{1} \mathrm{H}$ NMR.

It is noteworthy that we observed different outcomes for the scandium-catalyzed ring opening of styrene oxide (15), where both regioisomers were formed (the model reaction, Scheme 2) and 2-oxiranyl-pyridines (Schemes 3 and 4), where only $\beta$-amino alcohols were obtained. The observed nucleophilic attack at the benzylic $\beta$-position (regioselectivity of aminolysis) can be explained by the specific interaction of scandium ion complexed to the pyridine nitrogen and oxiranyl oxygen atoms, thus supporting the formation of both diastereomers of one regioisomer 21 (Figure 1). This is corroborated by the results of DFT calculations for the simplified models of trans-11 and its $\mathrm{Sc}^{3+}$ complex. The calculations indicated an increase of the length of $\mathrm{C} \beta$-epoxide oxygen bond and a substantial rise of the $\mathrm{C} \beta$ positive charge as measured by ESP (electrostatic potential charge) (see supporting file S2). It should be noted that the 


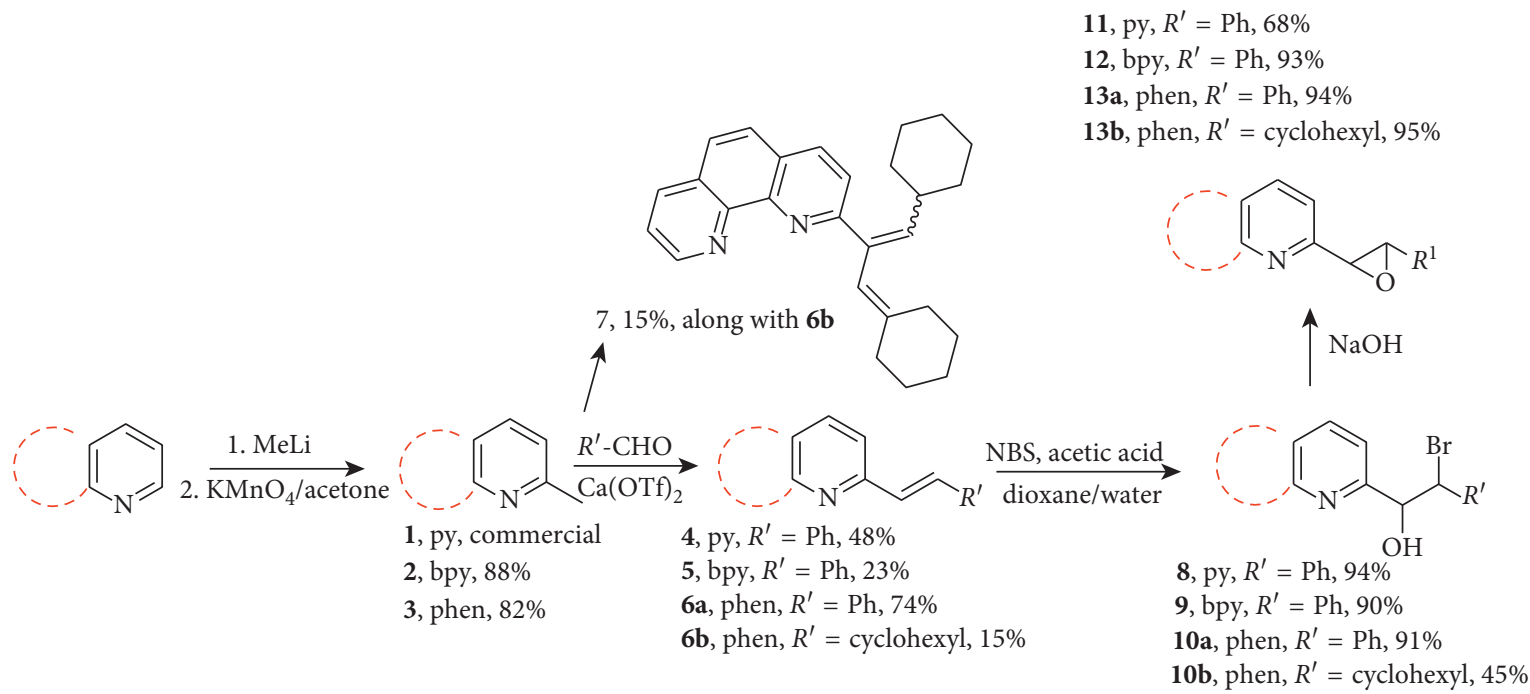

Scheme 1: Preparation of the $\alpha$-azaarene epoxides.
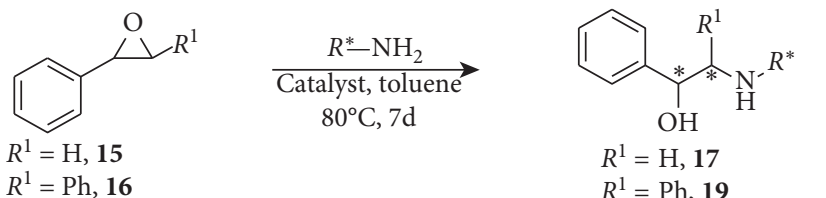<smiles>[R]N[C](CO)c1ccccc1</smiles>

$R^{1}=\mathrm{Ph}, 19$

$$
R^{*} \text {-NH2 }
$$

Epoxide Catalyst

Product, isolated yield (\%)

(S)-1-Phenylethylamine

$15 \mathrm{Sc}(\mathrm{OTf})_{3}$, DIEA

$\left(S, 1^{\prime} S\right)-\mathbf{1 7}, 19 \quad\left(R, 1^{\prime} S\right)-17,19$

$\left(R / S, 1^{\prime} S\right)-\mathbf{1 8}, 38$

(S)-1-Phenylethylamine

16

$\mathrm{Sc}(\mathrm{OTf})_{3}$, DIEA

$\left(1 S, 2 R, 1^{\prime} S\right)-19,27$

$\left.R, 2 S, 1^{\prime} S\right)-19,27$

(R)-1-Phenylethylamine

(S)-1-Phenylethylamine

$15 \mathrm{Zn}(\mathrm{OAc})_{2}$

$\left(R, 1^{\prime} R\right)-17,34$

$\left(S, 1^{\prime} R\right)-17,34$

(S)-1-Phenylethylamine

$\mathrm{Zn}(\mathrm{OAc})_{2}$

$\left(S, 1^{\prime} S\right)-\mathbf{1 7}, 34 \quad\left(R, 1^{\prime} S\right)-\mathbf{1 7}, 34$

15 No catalyst

$\left(S, 1^{\prime} S\right)-17$ and $\left(R, 1^{\prime} S\right)-17$ and $\left(R / S, 1^{\prime} S\right)-18$ (total $\left.4 \%\right)$

Scheme 2: Ring opening of styrene and stilbene oxides with chiral 1-phenylethylamine.<smiles>c1ccc(C2CO2)nc1</smiles>

14

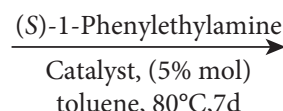

toluene, $80^{\circ} \mathrm{C}, 7 \mathrm{~d}$

Catalyst
$\mathrm{Zn}(\mathrm{OAc})_{2}$
$\mathrm{Sc}(\mathrm{OTf})_{3}$, DIEA

No catalyst<smiles>C[C@H](NC[C@H](O)c1ccccn1)c1ccccc1</smiles><smiles>C[C@H](NC[C@H](O)c1ccccn1)c1ccccc1</smiles>

Product, isolated yield (\%)

$\left(S, 1^{\prime} S\right)-\mathbf{2 0}, 17 \quad\left(R, 1^{\prime} S\right)-\mathbf{2 0}, 17 \quad($ total 34$)$

$\left(S, 1^{\prime} S\right)-\mathbf{2 0}, 36 \quad\left(R, 1^{\prime} S\right)-\mathbf{2 0}, 36 \quad($ total 72)

$\left(S, 1^{\prime} S\right)-20$ and $\left(R, 1^{\prime} S\right)-2(1: 1$ by NMR, total 5$)$

Scheme 3: Ring opening of 2-(oxiranyl)pyridine with (S)-1-phenylethylamine.

epoxide 11 has already been opened in the $\mathrm{MgBr}_{2}$-supported reaction with the same regioselectivity. That result was explained by similar $\mathrm{Mg}^{2+}$ complexation [52].

In order to confirm the configurations of obtained new ring-opening products, we transformed the amino alcohols into their cyclic urethanes (Scheme 5).

The respective ${ }^{1} \mathrm{H}$ NMR spectral patterns are very similar for the known (4R,5S,1'S)-26 [58] and new (4R,5R,1'S)-27. Their spectra substantially differ from that for $\left(4 S, 5 S, 1^{\prime} S\right)-27$
(Figure 2) (different configuration descriptors at C5 for $\left(4 R, 5 R, 1^{\prime} S\right)$-27 and $\left(4 R, 5 S, 1^{\prime} S\right)$-26 arise from CIP rules).

The obtained enantiomerically pure pyridine- $\beta$-amino alcohols: $\left(S, 1^{\prime} S\right)-\mathbf{2 0}, \quad\left(1 S, 2 S, 1^{\prime} R\right)-\mathbf{2 1}$, and $\left(1 S, 2 S, 1^{\prime} S\right)-\mathbf{2 1}$ and bipyridine- $\beta$-amino alcohols: $\left(1 S, 2 S, 1^{\prime} S\right)-\mathbf{2 3}$ and $\left(1 R, 2 R, 1^{\prime} S\right)-\mathbf{2 3}$ were preliminarily assessed as chiral catalysts in the asymmetric aldol reaction [62] of $p$-nitrobenzaldehyde with cyclohexanone. The reaction conditions were optimized by screening chiral ligands and metal salts. The highest selectivity for the anti-aldol 


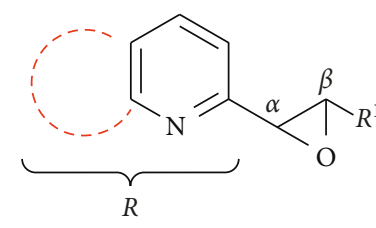

11-13

Epoxide

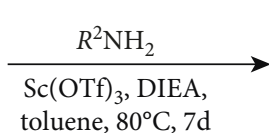

toluene, $80^{\circ} \mathrm{C}, 7 \mathrm{~d}$

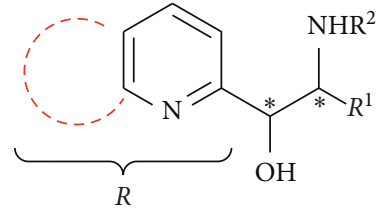

21-25

\begin{tabular}{|c|c|c|c|c|}
\hline \multicolumn{3}{|l|}{ Epoxide } & Chiral amine & Product \\
\hline$R=$ pyrid $-2-y l$ & $R^{1}=\mathrm{Ph}$ & 11 & $R^{2}=1$-phenylethyl & 21 \\
\hline$R=$ pyrid $-2-y l$ & $R^{1}=\mathrm{Ph}$ & 11 & $R^{2}=1$-cyclohexylethyl & 22 \\
\hline$R=2,2^{\prime}$-bipyrid-6-yl & $R^{1}=\mathrm{Ph}$ & 12 & $R^{2}=1$-phenylethyl & 23 \\
\hline$R=2,2^{\prime}$-bipyrid-6-yl & $R^{1}=\mathrm{Ph}$ & 12 & $R^{2}=1$-cyclohexylethyl & 24 \\
\hline$R=1,10$-phenanthrolin-2-yl, & $R^{1}=\mathrm{cycl}$ & $x y l, 13 b$ & $R^{2}=1$-phenylethyl & 25 \\
\hline
\end{tabular}

SCHEME 4: Ring opening of 2-oxiranyl-azaarenes with chiral amines.

TABLE 1: Ring opening of 2-oxiranyl-azaarenes with chiral amines.

\begin{tabular}{|c|c|c|c|}
\hline Epoxide & Amine & Product, yield $(\%)^{\mathrm{a}}$ & Total yield (\%) \\
\hline 11 & (S)-1-Phenylethylamine & $\left(1 S, 2 S, 1^{\prime} S\right)-21,26\left(1 R, 2 R, 1^{\prime} S\right)-21,26$ & 52 \\
\hline 11 & (R)-1-Phenylethylamine & $\left(1 R, 2 R, 1^{\prime} R\right)-\mathbf{2 1}, 29\left(1 S, 2 S, 1^{\prime} R\right)-\mathbf{2 1}, 29$ & 58 \\
\hline 11 & (R)-1-Cyclohexylethylamine & $\left(1 R, 2 R, 1^{\prime} R\right)-22,33\left(1 S, 2 S, 1^{\prime} R\right)-22,33$ & 66 \\
\hline 12 & (S)-1-Phenylethylamine & $\left(1 S, 2 S, 1^{\prime} S\right)-\mathbf{2 3}, 30\left(1 R, 2 R, 1^{\prime} S\right)-\mathbf{2 3}, 30$ & 60 \\
\hline 12 & (R)-1-Cyclohexylethylamine & $\left(1 R, 2 R, 1^{\prime} R\right)-24,32\left(1 S, 2 S, 1^{\prime} R\right)-24^{\mathrm{b}}$ & 64 \\
\hline $13 b$ & (R)-1-Phenylethylamine & $\left(1 R, 2 R, 1^{\prime} R\right)-25,\left(1 S, 2 S, 1^{\prime} R\right)-25^{c}$ & 8 \\
\hline
\end{tabular}

${ }^{a}$ The yield of each isolated diastereomer. ${ }^{b}$ Pure $\left(1 S, 2 S, 1^{\prime} R\right)-\mathbf{2 4}$ could not be isolated (remained in a mixture). ${ }^{c}$ The isolated $1: 1$ diastereomeric mixture was identified by HR-MS and ${ }^{1} \mathrm{H}$ NMR.
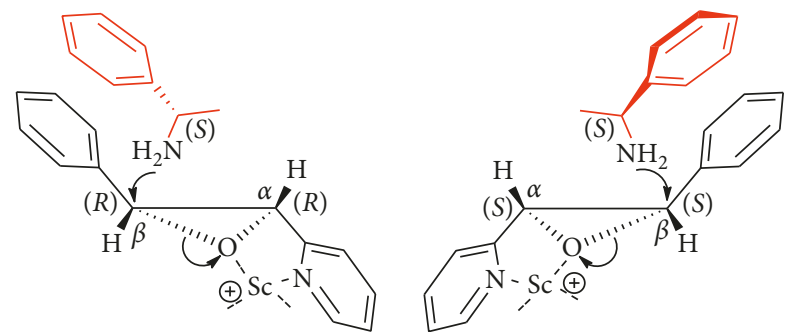

Figure 1: Models for regioselective aminolysis of rac-trans-11.<smiles>[R]C(O)C(NC([X])([R])c1ccccc1)c1ccccc1</smiles><smiles>CC(Cl)(Cl)OC(=O)OC(=O)O[Mg]</smiles>

$\left(1 S, 2 R, 1^{\prime} S\right)-19$

$\left(1 S, 2 S, 1^{\prime} S\right)-21$

$$
R=\mathrm{Ph}
$$

$\left(1 R, 2 R, 1^{\prime} S\right)-21$

$$
R=\text { pyridin }-2-y l
$$$$
R=\text { pyridin }-2-y l
$$

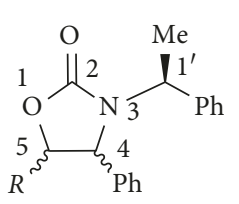

$\left(4 R, 5 S, 1^{\prime} S\right)-26 \quad 59 \%$ $\left(4 S, 5 S, 1^{\prime} S\right)-27 \quad 46 \%$ $\left(4 R, 5 R, 1^{\prime} S\right)-27 \quad 46 \%$
Scheme 5: Synthesis of oxazolidinones from $\beta$-amino alcohols.

$\left(2 S, 1^{\prime} R\right) \quad 55 \%$ ee and $38 \%$ conversion was obtained with $\left(1 R, 2 R, 1^{\prime} S\right)-23$ and $\mathrm{Zn}(\mathrm{OAc})_{2}+\mathrm{HOAc}$. For the details, see supporting file $\mathrm{S} 3$.
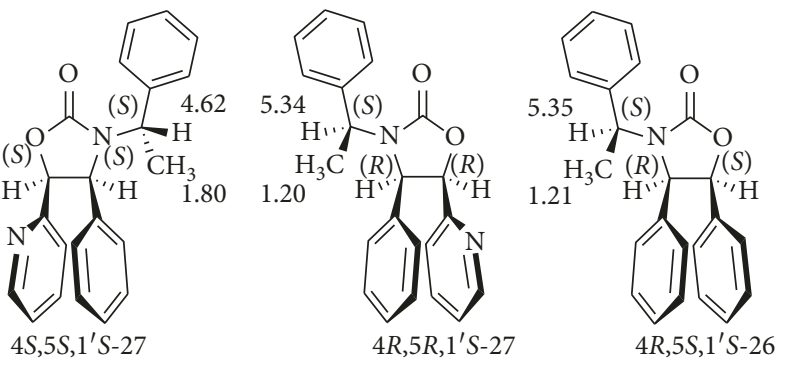

Figure 2: Structures and selected ${ }^{1} \mathrm{H}$ NMR $\left(400 \mathrm{MHz}, \mathrm{CDCl}_{3}\right)$ resonances of oxazolidinones 26 and 27.

\section{Conclusions}

Concluding, we have developed an efficient synthesis of 2oxiranyl-azaarenes designed as precursors of chiral ligands and synthetic building blocks. The regioselective epoxide ring opening with chiral primary amines in the presence of $\mathrm{Sc}(\mathrm{OTf})_{3}$ and DIEA gave the corresponding $\beta$-amino alcohols, derivatives of pyridine and $2,2^{\prime}$-bipyridine. The resulting diastereomeric compounds were separated, and their stereochemical configurations were proved by correlation with the known analogs. The enantiomerically pure pyridine- $\beta$-amino alcohol was preliminarily tested as chiral ligands in the asymmetric aldol reaction with up to $55 \%$ ee outcome. 


\section{Experimental}

4.1. General Information. Solvents were distilled, and other reagents were used as received. Reactions were monitored by thin-layer chromatography (TLC) on silica gel $60 \mathrm{~F}-254$ or aluminum oxide F-25 (Type E) precoated plates, and spots were visualized with a UV lamp and/or Dragendorff reagent. Separation of products by chromatography was carried out on silica gel 60 (230-400 mesh) or aluminum oxide (neutral). Melting points were determined using an Electrothermal IA 91100 digital melting-point apparatus using the standard open capillary method and are uncorrected. Observed rotations at $589 \mathrm{~nm}$ were measured using an Optical Activity Ltd. Model AA-5 automatic polarimeter. ${ }^{1} \mathrm{H}$ and ${ }^{13} \mathrm{C}$ NMR spectra $(400,600 \mathrm{MHz}$ and $100,151 \mathrm{MHz}$, respectively) were collected on Jeol 400yh and Bruker Avance II 600 instruments. The spectra were recorded in $\mathrm{CDCl}_{3}$ referenced to the respective residual signals of the solvent. Chemical shifts are given in parts per million (ppm) downfield from tetramethylsilane as the internal standard in a deuterated solvent and coupling constants $(J)$ are in Hertz $(\mathrm{Hz})$. Infrared spectra $\left(4000-400 \mathrm{~cm}^{-1}\right)$ were collected on a Fourier transform, Bruker VERTEX 70V spectrometer using diamond ATR accessory. High-resolution mass spectra were recorded using electrospray ionization on Waters LCT Premier XE TOF instrument.

\subsection{Synthesis of Methyl Derivatives}

4.2.1. 6-Methyl-2,2'-bipyridine 2. The methylation was performed according to the literature procedure [47]. Brown oil, $7.6 \mathrm{~g}, 88 \%$ yield, $R_{\mathrm{f}}=0.56\left(\mathrm{CHCl}_{3}: \mathrm{AcOEt}: \mathrm{MeOH} 1: 1\right.$ : 0.25). ${ }^{1} \mathrm{H}$ NMR $\left(600 \mathrm{MHz}, \mathrm{CDCl}_{3}\right) \delta: 8.59-8.58(\mathrm{~m}, 1 \mathrm{H})$, $8.35-8.33(\mathrm{~m}, 1 \mathrm{H}), 8.12(\mathrm{~d}, J=7.8 \mathrm{~Hz}, 1 \mathrm{H}), 7.69-7.66(\mathrm{~m}$, $1 \mathrm{H}), 7.59-7.56(\mathrm{~m}, 1 \mathrm{H}), 7.17-7.15(\mathrm{~m}, 1 \mathrm{H}), 7.04(\mathrm{~d}$, $J=7.8 \mathrm{~Hz}, 1 \mathrm{H}), 2.54(\mathrm{~s}, 3 \mathrm{H})$. The NMR data are in agreement with the reported ones [47].

4.2.2. 2-Methyl-1,10-phenanthroline 3. The methylation was performed according to a modified literature procedure [48]. A solution of methyllithium in diethyl ether $(1.6 \mathrm{M}, 45 \mathrm{~mL}$, $72 \mathrm{mmol}$ ) was added dropwise to a solution of 1,10-phenanthroline $(10 \mathrm{~g}, 55 \mathrm{mmol})$ in toluene $(200 \mathrm{~mL})$ at $-72^{\circ} \mathrm{C}$ under $\mathrm{Ar}$ atmosphere. The reaction mixture was stirred for $3 \mathrm{~h}$ at $-72^{\circ} \mathrm{C}$ and for $2 \mathrm{~h}$ at room temperature. Then, ice was added with stirring in an ice-water bath and the resulting solution turned red. The aqueous layer was separated and extracted with diethyl ether $(3 \times 50 \mathrm{~mL})$. The combined organic phases were washed twice with brine and dried over $\mathrm{Na}_{2} \mathrm{SO}_{4}$, and ether was removed. The resulting orange toluene solution was treated with $\mathrm{MnO}_{2}(54 \mathrm{~g})$, stirred for $24 \mathrm{~h}$, and then filtered through Celite. The solvent was removed in vacuo to give a crude product. Column chromatography on neutral alumina with $t$-butyl methyl ether (MTBE) as an eluent gave pure $3(8.73 \mathrm{~g} 82 \%)$, as yellow crystals, m.p. $76-77^{\circ} \mathrm{C}$ (lit. [48] m.p. $\left.75-76^{\circ} \mathrm{C}\right) .{ }^{1} \mathrm{H}$ NMR $(600 \mathrm{MHz}$, $\left.\mathrm{CDCl}_{3}\right) \delta: 9.17(\mathrm{dd}, J=4.2,1.8 \mathrm{~Hz}, 1 \mathrm{H}), 8.18(\mathrm{dd}, J=8.0$, $1.8 \mathrm{~Hz}, 1 \mathrm{H}), 8.08(\mathrm{~d}, J=8.2 \mathrm{~Hz}, 1 \mathrm{H}), 7.69(\mathrm{q}, J=8.8 \mathrm{~Hz}, 2 \mathrm{H})$, $7.56(\mathrm{dd}, J=8.2,4.6 \mathrm{~Hz}, 1 \mathrm{H}), 7.47(\mathrm{~d}, J=8.2 \mathrm{~Hz}, 1 \mathrm{H}), 2.92$ $(\mathrm{s}, 3 \mathrm{H})$. The NMR data are in agreement with the reported ones [48].

4.3. General Procedure for the Synthesis of 2-Styryl-azaarenes. The synthesis of 2-styryl-azaarenes was performed according to a modified literature procedure [50]. The mixture of 2methylazaarene $(1 \mathrm{mmol})$, benzaldehyde $(1 \mathrm{mmol})$, and $\mathrm{Ca}(\mathrm{OTf})_{2}$ [49] (5 mol\%) was heated at $120^{\circ} \mathrm{C}$ under argon atmosphere for $48 \mathrm{~h}$ for $\alpha$-picoline or $96 \mathrm{~h}$ for derivatives of bipyridine and phenanthroline. After the reaction completion (monitored by TLC), the mixture was dissolved in $1 \mathrm{M}$ $\mathrm{HCl}$ and extracted with diethyl ether $(3 \times 15 \mathrm{~mL})$. The remaining aqueous layer was alkalized with $\mathrm{NaOH}$, extracted with diethyl ether $(3 \times 25 \mathrm{~mL})$, dried over $\mathrm{Na}_{2} \mathrm{SO}_{4}$, and concentrated in vacuo to give crude product.

4.3.1. 2-[(E)-2-Phenylethenyl]pyridine (4). White crystals, $88 \mathrm{mg}, 48 \%$ yield, recrystallized $\left(\mathrm{CH}_{2} \mathrm{Cl}_{2} /\right.$ hexane), m.p. 89-90 ${ }^{\circ} \mathrm{C}$ (lit. [51] m.p. $\left.89-91^{\circ} \mathrm{C}\right), R_{\mathrm{f}}=0.67\left(\mathrm{CHCl}_{3}: \mathrm{AcOEt}\right.$ : $\mathrm{MeOH} 1: 1: 0.25) .{ }^{1} \mathrm{H}$ NMR $\left(600 \mathrm{MHz}, \mathrm{CDCl}_{3}\right) \delta: 8.62-8.61$ (m, 1H), 7.68-7.63 (m, 1H), 7.59-7.58 (m, 2H), 7.41-7.37 (m, 3H), 7.32-7.29 (m, 1H), 7.20 (s, 1H), 7.17-7.15 (m, 2H). The NMR data are in agreement with the reported ones [51].

4.3.2. 6-[(E)-2-Phenylethenyl]-2,2'-bipyridine (5). White crystals, $60 \mathrm{mg}, 23 \%$ yield, recrystallized $\left(\mathrm{CH}_{2} \mathrm{Cl}_{2} /\right.$ hexane), m.p. $117-118^{\circ} \mathrm{C}, R_{\mathrm{f}}=0.70 \quad\left(\mathrm{CHCl}_{3}: \mathrm{AcOEt}: \mathrm{MeOH} 1: 1\right.$ : 0.25). ${ }^{1} \mathrm{H}$ NMR $\left(600 \mathrm{MHz}, \mathrm{CDCl}_{3}\right) \delta: 8.71(\mathrm{~d}, J=6.0 \mathrm{~Hz}, 1 \mathrm{H})$, $8.59(\mathrm{~d}, J=6.0 \mathrm{~Hz}, 1 \mathrm{H}), 8.30(\mathrm{~d}, J=7.8 \mathrm{~Hz}, 1 \mathrm{H}), 7.89-7.86(\mathrm{~m}$, $1 \mathrm{H}), 7.82-7.77(\mathrm{~m}, 2 \mathrm{H}), 7.64(\mathrm{~d}, J=7.2 \mathrm{~Hz}, 2 \mathrm{H}), 7.42-7.39$ $(\mathrm{m}, 3 \mathrm{H}), 7.35-7.30(\mathrm{~m}, 2 \mathrm{H}) 7.28-7.25(\mathrm{~m}, 1 \mathrm{H}) ;{ }^{13} \mathrm{C}$ NMR $\left(600 \mathrm{MHz}, \mathrm{CDCl}_{3}\right) \delta: 156.2,155.6,155.0,148.9,137.5,137.1$, $136.8,132.9,128.7,128.3,128.2,127.2,123.8,122.2,121.4$, 119.6; HR-MS (ESI) $\left[\mathrm{C}_{18} \mathrm{H}_{14} \mathrm{~N}_{2}+\mathrm{H}\right]^{+}$requires 259.1230; found 259.1223 .

4.3.3. 2-[(E)-2-Phenylethenyl]-1,10-phenanthroline (6a). Brown oil, $210 \mathrm{mg}, 74 \%$ yield, purified by column chromatography $\left(\mathrm{SiO}_{2}, \mathrm{CHCl}_{3}: \mathrm{AcOEt}: \mathrm{MeOH} \quad 1: 1: 0.25\right)$, $R_{\mathrm{f}}=0.62 \quad\left(\mathrm{CHCl}_{3}: \mathrm{AcOEt}: \mathrm{MeOH} \quad 1: 1: 0.25\right) .{ }^{1} \mathrm{H} \quad \mathrm{NMR}$ $\left(600 \mathrm{MHz}, \mathrm{CDCl}_{3}\right) \delta: 9.28(\mathrm{dd}, J=4.3,1.8 \mathrm{~Hz}, 1 \mathrm{H}), 8.29(\mathrm{dd}$, $J=8.2,1.8 \mathrm{~Hz}, 1 \mathrm{H}), 8.21(\mathrm{~d}, J=8.2 \mathrm{~Hz}, 1 \mathrm{H}), 7.95$ (d, $J=6.0 \mathrm{~Hz}, 1 \mathrm{H}), 7.86-7.78(\mathrm{~m}, 3 \mathrm{H}), 7.76-7.72(\mathrm{~m}, 1 \mathrm{H}), 7.75$ $(\mathrm{d}, J=6.0 \mathrm{~Hz}, 2 \mathrm{H}), 7.67-7.65(\mathrm{~m}, 1 \mathrm{H}), 7.42-7.40(\mathrm{~m}, 2 \mathrm{H})$ 7.34-7.31 (m, 1H); ${ }^{13} \mathrm{C}$ NMR $\left(600 \mathrm{MHz}, \mathrm{CDCl}_{3}\right) \delta: 156.6$, $149.9,145.5,145.4,136.8,136.6,136.4,134.8,129.5,129.1$, 128.8, 128.6, 127.6, 127.4, 126.7, 125.8, 122.9, 120.7; HR-MS (ESI) $\left[\mathrm{C}_{20} \mathrm{H}_{14} \mathrm{~N}_{2}+\mathrm{H}\right]^{+}$requires 283.1230; found 283.1242.

4.3.4. 2-[(E)-2-Cyclohexylethenyl)-1,10-phenanthroline (6b). Yellow oil, $43 \mathrm{mg}, 15 \%$ yield, purified by column chromatography $\left(\mathrm{Al}_{2} \mathrm{O}_{3}, 20 \% \mathrm{AcOEt} /\right.$ hexane $) .{ }^{1} \mathrm{H}$ NMR $(400 \mathrm{MHz}$, $\left.\mathrm{CDCl}_{3}\right) \delta: 9.20(\mathrm{dd}, J=4.6,1.8 \mathrm{~Hz}, 1 \mathrm{H}), 8.22(\mathrm{dd}, J=8.2$, $1.8 \mathrm{~Hz}, 1 \mathrm{H}), 8.13(\mathrm{~d}, J=8.2 \mathrm{~Hz}, 1 \mathrm{H}), 7.80(\mathrm{~d}, J=8.2 \mathrm{~Hz}, 1 \mathrm{H})$, $7.72(\mathrm{q}, J=8.9 \mathrm{~Hz}, 2 \mathrm{H}), 7.60(\mathrm{dd}, J=8.2,4.6 \mathrm{~Hz}, 1 \mathrm{H}), 7.01$ 
(dd, $J=16.2,1.2 \mathrm{~Hz}, 1 \mathrm{H}), 6.81(\mathrm{dd}, J=16.2,6.4 \mathrm{~Hz}, 1 \mathrm{H})$, $2.30-2.24(\mathrm{~m}, 1 \mathrm{H}), 1.92-1.88(\mathrm{~m}, 2 \mathrm{H}), 1.82-1.77(\mathrm{~m}, 2 \mathrm{H})$, $1.72-1.66(\mathrm{~m}, 1 \mathrm{H}), 1.41-1.16(\mathrm{~m}, 5 \mathrm{H}) ;{ }^{13} \mathrm{C} \mathrm{NMR}(400 \mathrm{MHz}$, $\left.\mathrm{CDCl}_{3}\right) \delta: 157.4,150.3,146.2,143.3,136.3,136.2,129.6$, $128.9,127.3,126.6,126.5,125.7,122.8,119.9,41.2,32.6,26.3$, 26.2; HR-MS (ESI) $\left[\mathrm{C}_{20} \mathrm{H}_{20} \mathrm{~N}_{2}+\mathrm{H}\right]^{+}$requires 289.1699; found 289.1705 .

4.3.5. 1-Cyclohexyl-3-cyclohexylidene-2-(1,10-phenonthrolin$2-y l$ )propene (7). Yellow oil, $42 \mathrm{mg}, 15 \%$ yield, purified by column chromatography $\left(\mathrm{Al}_{2} \mathrm{O}_{3}, 20 \%\right.$ AcOEt/hexane $) .{ }^{1} \mathrm{H}$ $\operatorname{NMR}\left(400 \mathrm{MHz}, \mathrm{CDCl}_{3}\right) \delta: 9.17(\mathrm{dd}, J=4.3,1.5 \mathrm{~Hz}, 1 \mathrm{H}), 8.20$ (dd, $J=7.9,1.8 \mathrm{~Hz}, 1 \mathrm{H}), 8.09(\mathrm{~d}, J=8.2 \mathrm{~Hz}, 1 \mathrm{H}), 7.73-7.66$ $(\mathrm{m}, 3 \mathrm{H}), 7.59(\mathrm{dd}, J=7.9,4.3 \mathrm{~Hz}, 1 \mathrm{H}), 7.02(\mathrm{dd}, J=9.8$, $1.5 \mathrm{~Hz}, 1 \mathrm{H}), 6.05(\mathrm{~s}, 1 \mathrm{H}), 2.46-2.43(\mathrm{~m}, 1 \mathrm{H}), 2.34-2.31(\mathrm{~m}$, 2H), 1.96-1.93 (m, 2H), 1.77-1.75 (m, 4H), 1.69-1.63 (m, $3 \mathrm{H}), \quad 1.55-1.50(\mathrm{~m}, 2 \mathrm{H}), 1.40-1.31(\mathrm{~m}, 7 \mathrm{H}) ;{ }^{13} \mathrm{C} \mathrm{NMR}$ $\left(400 \mathrm{MHz}, \mathrm{CDCl}_{3}\right) \delta: 159.4,150.1,146.4,145.8,144.9,141.4$, $136.11,136.09,134.6,129.0,127.3,126.6,125.4,122.7,121.9$, $118.3,39.1,36.9,32.4,30.6,28.8,27.3,26.7,26.3,26.2$; HRMS (ESI) $\quad\left[\mathrm{C}_{27} \mathrm{H}_{30} \mathrm{~N}_{2}+\mathrm{H}\right]^{+}$requires 383.2482; found 383.2483 .

4.4. General Procedure for the Synthesis of Bromohydrins. The synthesis of bromohydrins was performed according to a modified literature procedure [53]. The mixture of 2-styrylazaarene $(3.0 \mathrm{mmol})$, NBS $(587 \mathrm{mg}, 3.3 \mathrm{mmol})$, and acetic acid $(0.5 \mathrm{~mL})$ was dissolved in the mixture of dioxane/water $(3.5 \mathrm{~mL} / 7 \mathrm{~mL})$ and stirred at room temperature for $24 \mathrm{~h}$. After completion of the reaction, the mixture was extracted with chloroform $(3 \times 30 \mathrm{~mL})$. The combined organic layers were dried by over $\mathrm{Na}_{2} \mathrm{SO}_{4}$ filtered and concentrated in vacuo to give the desired product as confirmed by ${ }^{1} \mathrm{H}$ NMR.

4.4.1. 2-Bromo-1-phenyl-2-(pyridin-2-yl)ethanol (8). Brown oil, $784 \mathrm{mg}$, 94\% yield; $R_{\mathrm{f}}=0.57\left(\mathrm{CHCl}_{3}: \mathrm{AcOEt}: \mathrm{MeOH} 1\right.$ : $1: 0.25) .{ }^{1} \mathrm{H}$ NMR $\left(600 \mathrm{MHz}, \mathrm{CDCl}_{3}\right) \delta: 8.57-8.56(\mathrm{~m}, 1 \mathrm{H})$, $7.63-7.60(\mathrm{~m}, 2 \mathrm{H}), 7.30-7.27(\mathrm{~m}, 2 \mathrm{H}), 7.27-7.25(\mathrm{~m}, 2 \mathrm{H})$, 7.23-7.21 (m, 2H), $5.41(\mathrm{~d}, J=5.4 \mathrm{~Hz}, 1 \mathrm{H}), 5.20(\mathrm{~d}, J=5.4 \mathrm{~Hz}$, $1 \mathrm{H})$.

4.4.2. 2-Bromo-1-phenyl-2-(2,2'-bipyridin-6-yl)ethanol (9). White crystals, $960 \mathrm{mg}, 90 \%$ yield, purified by column chromatography $\left(\mathrm{SiO}_{2}, \mathrm{CHCl}_{3}\right.$ : AcOEt: $\left.\mathrm{MeOH} 1: 1: 0.25\right)$, m.p. $117-118^{\circ} \mathrm{C}, R_{\mathrm{f}}=0.61 \quad\left(\mathrm{CHCl}_{3}: \mathrm{AcOEt}: \mathrm{MeOH} 1: 1\right.$ : 0.25). ${ }^{1} \mathrm{H}$ NMR $\left(600 \mathrm{MHz}, \mathrm{CDCl}_{3}\right) \delta: 8.72(\mathrm{~d}, J=4.8 \mathrm{~Hz}, 1 \mathrm{H})$, 8.38-8.34 (m, 1H), $7.90(\mathrm{~s}, 1 \mathrm{H}), 7.77-7.74(\mathrm{t}, J=7.8 \mathrm{~Hz}, 1 \mathrm{H})$, $7.40-7.38(\mathrm{~m}, 1 \mathrm{H}), 7.32(\mathrm{~d}, J=7.2 \mathrm{~Hz}, 1 \mathrm{H}) 7.28-7.24(\mathrm{~m}$, $4 \mathrm{H}), 7.24-7.22(\mathrm{~m}, 2 \mathrm{H}), 5.53(\mathrm{~d}, J=5.4 \mathrm{~Hz}, 1 \mathrm{H}), 5.28(\mathrm{~d}$, $J=5.4 \mathrm{~Hz}, 1 \mathrm{H})$.

4.4.3. 2-Bromo-1-phenyl-2-(1,10-phenanthrolin-2-yl)ethanol (10a). Brown oil, $1.0 \mathrm{~g}$, 91\% yield, purified by column chromatography $\left(\mathrm{SiO}_{2}, \mathrm{CHCl}_{3}\right.$ : AcOEt: $\left.\mathrm{MeOH} 1: 1: 0.25\right)$, $R_{\mathrm{f}}=0.24 \quad\left(\mathrm{CHCl}_{3}:\right.$ AcOEt $\left.: \mathrm{MeOH} 1: 1: 0.25\right) .{ }^{1} \mathrm{H} \quad \mathrm{NMR}$ $\left(600 \mathrm{MHz}, \mathrm{CDCl}_{3}\right) \delta: 9.31-9.30(\mathrm{~m}, 1 \mathrm{H}), 8.40(\mathrm{~d}, J=7.8 \mathrm{~Hz}$,
$1 \mathrm{H}), 8.24(\mathrm{~d}, J=8.4 \mathrm{~Hz}, 1 \mathrm{H}), 7.87-7.83(\mathrm{~m}, 2 \mathrm{H}), 7.78-7.75$ (m, 2H), 7.55 (d, J=7.2 Hz, 2H), 7.29-7.27 (m, 2H), 7.24$7.21(\mathrm{~m}, 1 \mathrm{H}), 5.79(\mathrm{~d}, J=6.6 \mathrm{~Hz}, 1 \mathrm{H}), 5.60(\mathrm{~d}, J=6.6 \mathrm{~Hz}, 1 \mathrm{H})$.

4.4.4. 2-Bromo-1-cyclohexyl-2-(1,10-phenanthrolin-2-yl)ethanol (10b). Yellow oil, $520 \mathrm{mg}, 45 \%$ yield, purified by column chromatography $\left(\mathrm{Al}_{2} \mathrm{O}_{3}, \mathrm{CHCl}_{3}\right.$ : AcOEt: hexane $1: 1$ : 2). ${ }^{1} \mathrm{H} \mathrm{NMR}\left(400 \mathrm{MHz}, \mathrm{CDCl}_{3}\right) \delta: 9.15(\mathrm{dd}, J=4.3,1.8 \mathrm{~Hz}$, $1 \mathrm{H}), 8.25(\mathrm{dd}, J=8.2,1.8 \mathrm{~Hz}, 1 \mathrm{H}), 8.20(\mathrm{~d}, J=8.2 \mathrm{~Hz}, 1 \mathrm{H})$, 7.81-7.75 (m, 3H), $7.63(\mathrm{dd}, J=8.2,4.6 \mathrm{~Hz}, 1 \mathrm{H}), 5.17$ (d, $J=9.2 \mathrm{~Hz}, 1 \mathrm{H}), 4.36(\mathrm{dd}, J=9.5,2.4 \mathrm{~Hz}, 1 \mathrm{H}), 2.57(\mathrm{~s}, 2 \mathrm{H})$, 2.27-2.25 (m, 1H), 2.04-2.02 (m, 1H), 1.82-1.16 (m, 7H).

4.5. General Procedure for the Synthesis of Epoxides. The synthesis of epoxides was performed according to a modified literature procedure [53]. Bromohydrin $(2.8 \mathrm{mmol})$ was dissolved in dioxane $(3.5 \mathrm{~mL})$, then $1 \mathrm{M}$ aqueous $\mathrm{NaOH}$ $(4.5 \mathrm{~mL})$ was added, and the mixture was stirred at room temperature for $24 \mathrm{~h}$. After completion of the reaction, the mixture was extracted with chloroform $(3 \times 30 \mathrm{~mL})$. The combined organic layers were dried over $\mathrm{Na}_{2} \mathrm{SO}_{4}$, filtered, and concentrated in vacuo to give the desired product.

4.5.1. trans-2-(3-Phenyl-2-oxiranyl)pyridine (11). Brown oil, $375 \mathrm{mg}, 68 \%$ yield, $R_{\mathrm{f}}=0.63\left(\mathrm{CHCl}_{3}:\right.$ AcOEt $: \mathrm{MeOH} 1: 1$ : 0.25). ${ }^{1} \mathrm{H}$ NMR $\left(600 \mathrm{MHz}, \mathrm{CDCl}_{3}\right) \delta: 8.60-8.58(\mathrm{~m}, 1 \mathrm{H})$, 7.73-7.69 (m, 1H), 7.38-7.31 (m, 6H), 7.26-7.23 (m, 1H), 4.05 (AB system, 2H). The NMR data are in agreement with the reported ones [52].

4.5.2. trans-6-(3-Phenyl-2-oxiranyl)-2,2'-bipyridine (12). White crystals, $713 \mathrm{mg}, 93 \%$ yield, m.p. $117-118^{\circ} \mathrm{C}, R_{\mathrm{f}}=0.61$ $\left(\mathrm{CHCl}_{3}: \mathrm{AcOEt}: \mathrm{MeOH} 1: 1: 0.25\right)$. IR $v_{\max }$ (Neat) 2960, 2926, 1580, 1563, 775, 760, 746, $694 \mathrm{~cm}^{-1} ;{ }^{1} \mathrm{H} \mathrm{NMR}$ $\left(600 \mathrm{MHz}, \mathrm{CDCl}_{3}\right) \delta: 8.7(\mathrm{~d}, J=4.2 \mathrm{~Hz}, 1 \mathrm{H}), 8.47(\mathrm{~d}$, $J=7.8 \mathrm{~Hz}, 1 \mathrm{H}), 8.4(\mathrm{~d}, J=7.8 \mathrm{~Hz}, 1 \mathrm{H}), 7.88-7.84(\mathrm{~m}, 2 \mathrm{H})$, 7.40-7.36 (m, 4H), 7.35-7.26 (m, 3H), $4.16(\mathrm{~d}, J=1.8 \mathrm{~Hz}$, $1 \mathrm{H}), 4.12(\mathrm{~d}, J=1.8 \mathrm{~Hz}, 1 \mathrm{H}) ;{ }^{13} \mathrm{C} \mathrm{NMR}\left(600 \mathrm{MHz}, \mathrm{CDCl}_{3}\right) \delta$ : $156.1,155.8,155.7,149.1,137.8,137.0,136.8,128.6,128.5$, $125.8,123.9,121.4,120.6,119.7,63.2,61.9$; HR-MS (ESI) $\left[\mathrm{C}_{18} \mathrm{H}_{14} \mathrm{~N}_{2} \mathrm{O}+\mathrm{H}\right]^{+}$requires 275.1179; found 275.1180 .

4.5.3. trans-2-(3-Phenyl-2-oxiranyl)-1,10-phenanthroline (13a). Brown oil, $784 \mathrm{mg}, 94 \%$ yield, $R_{\mathrm{f}}=0.44\left(\mathrm{CHCl}_{3}\right.$ : AcOEt : MeOH $1: 1: 0.25)$. IR $v_{\max }$ (Neat) 2923, 2853, 1588, $1555,845,741,696 \mathrm{~cm}^{-1} ;{ }^{1} \mathrm{H} \mathrm{NMR}\left(600 \mathrm{MHz}, \mathrm{CDCl}_{3}\right) \delta: 9.18$ $(\mathrm{dd}, J=4.3,1.8 \mathrm{~Hz}, 1 \mathrm{H}), 8.31-8.24(\mathrm{~m}, 2 \mathrm{H}), 7.80(\mathrm{~d}$, $J=1.8 \mathrm{~Hz}, 2 \mathrm{H}), 7.63(\mathrm{dd}, J=8.2,6.4 \mathrm{~Hz}, 2 \mathrm{H}), 7.37-7.33(\mathrm{~m}$, $5 \mathrm{H}), 4.69(\mathrm{~d}, J=1.8 \mathrm{~Hz}, 1 \mathrm{H}), 4.02(\mathrm{~d}, J=1.8 \mathrm{~Hz} 1 \mathrm{H}) ;{ }^{13} \mathrm{C}$ NMR $\left(600 \mathrm{MHz}, \mathrm{CDCl}_{3}\right) \delta: 158.0,150.6,146.0,145.8,137.5$, $136.6,136.3,129.2,128.7,128.6,128.1,126.7,126.6,125.9$, 123.3, 118.1, 64.0, 63.1; HR-MS (ESI) $\left[\mathrm{C}_{20} \mathrm{H}_{14} \mathrm{~N}_{2} \mathrm{O}+\mathrm{H}\right]^{+}$ requires 299.1135; found 299.1146 .

4.5.4. trans-2-(3-Cyclohexyl-2-oxiranyl)-1,10-phenanthroline (13b). Yellow oil, $811 \mathrm{mg}$, 95\% yield. ${ }^{1} \mathrm{H}$ NMR $(400 \mathrm{MHz}$, 
$\left.\mathrm{CDCl}_{3}\right) \delta: 9.24(\mathrm{dd}, J=4.3,1.5 \mathrm{~Hz}, 1 \mathrm{H}), 8.26(\mathrm{dd}, J=8.2$, $1.8 \mathrm{~Hz}, 1 \mathrm{H}), 8.22(\mathrm{~d}, J=8.2 \mathrm{~Hz}, 1 \mathrm{H}), 7.78$ (s, 2H), 7.65 (dd, $J=8.2,4.6 \mathrm{~Hz}, 1 \mathrm{H}), 7.45(\mathrm{~d}, J=8.2 \mathrm{~Hz}, 1 \mathrm{H}), 4.47$ (d, $J=2.1 \mathrm{~Hz}, 1 \mathrm{H}), 2.96(\mathrm{~d}, J=2.1 \mathrm{~Hz}, 1 \mathrm{H}), 1.59-1.26(\mathrm{~m}, 3 \mathrm{H})$, $1.25-1.24(\mathrm{~m}, 6 \mathrm{H}), 0.86-0.84(\mathrm{~m}, 2 \mathrm{H}) ;{ }^{13} \mathrm{C} \mathrm{NMR}(600 \mathrm{MHz}$, $\left.\mathrm{CDCl}_{3}\right) \delta: 159.2,150.6,137.1,136.2,134.3,129.6,129.1$, 128.6, 126.6, 126.5, 123.1, 118.3, 38.9, 30.6, 24.1 23.0, 14.1, 11.2; HR-MS (ESI) $\left[\mathrm{C}_{20} \mathrm{H}_{20} \mathrm{~N}_{2} \mathrm{O}+\mathrm{H}\right]^{+}$requires 305.1648; found 305.1653 .

\subsection{Procedures for Ring Opening of Epoxides}

4.6.1. Method $A$ (Catalyzed by $\mathrm{Sc}(\mathrm{OTf})_{3}$ ). A solution of the epoxide $11,12,13 b, 14,15$, or $16(0.5 \mathrm{mmol})$, 1-phenylethylamine $(77 \mu \mathrm{L}, 0.6 \mathrm{mmol}), \mathrm{Sc}(\mathrm{OTf})_{3}(12 \mathrm{mg}, 5 \mathrm{~mol} \%)$, and $N$-ethyldiisopropylamine $(170 \mu \mathrm{L}, 1 \mathrm{mmol})$ in toluene $(2 \mathrm{~mL})$ was stirred under argon in a sealed test tube at $80^{\circ} \mathrm{C}$ for 7 days. The cooled mixture was directly submitted to the column chromatography on silica gel. In this way, the regioisomers 17 and $\mathbf{1 8}$, resulting in the reaction of 15 , were separated, and their structures were confirmed by NMR. The isolated diastereoisomers $\mathbf{1 7}$, as well as the respective diastereomers formed in the reactions of $11,12,14$, and 16, were separated by column chromatography. Additionally, the diastereomers 17 could be separated by recrystallization from hexane $/ \mathrm{CH}_{2} \mathrm{Cl}_{2}$.

The reactions of $11(98 \mathrm{mg}, 0.5 \mathrm{mmol})$ or $\mathbf{1 2}(137 \mathrm{mg}$, $0.5 \mathrm{mmol})$ with $(R)-1$-cyclohexylethylamine $(88 \mu \mathrm{L}, 0.6 \mathrm{mmol})$, $\mathrm{Sc}(\mathrm{OTf})_{3} \quad(12 \mathrm{mg}, 5 \mathrm{~mol} \%)$, and $N$-ethyldiisopropylamine $(170 \mu \mathrm{L}, 1 \mathrm{mmol})$ dissolved in toluene $(2 \mathrm{~mL})$ were run and then worked up as above. For the epoxide 11, both diastereomers were separated by chromatography and gave pure samples, while for $\mathbf{1 2}$, only one diastereomer $\left(1 R, 2 R, 1^{\prime} R\right)-\mathbf{2 4}$ could be isolated in a pure form.

The pure separated products $\left(S, 1^{\prime} S\right)-\mathbf{1 7},\left(R, 1^{\prime} S\right)-\mathbf{1 7},(S /$ $\left.R, 1^{\prime} S\right)-18,\left(1 R, 2 S, 1^{\prime} S\right)-19,\left(1 S, 2 R, 1^{\prime} S\right)-19,\left(S, 1^{\prime} S\right)-20,\left(R, 1^{\prime} S\right)-$ 20, $\left(1 R, 2 S, 1^{\prime} S\right)-\mathbf{2 1},\left(1 S, 2 S, 1^{\prime} S\right)-\mathbf{2 1},\left(1 S, 2 R, 1^{\prime} R\right)-\mathbf{1 9},\left(1 R, 2 R, 1^{\prime} R\right)-$ 21, $\left(1 S, 2 R, 1^{\prime} R\right)-22,\left(1 R, 2 R, 1^{\prime} R\right)-22,\left(1 R, 2 S, 1^{\prime} S\right)-23,\left(1 S, 2 S, 1^{\prime} S\right)-$ 23, $\left(1 R, 2 R, 1^{\prime} R\right)-24$, and $\left[\left(1 R, 2 R, 1^{\prime} R\right)\right.$ - and $\left.\left(1 S, 2 S, 1^{\prime} R\right)\right]-25$ were analyzed, and their properties are reported below.

4.6.2. Method $B$ (Catalyzed by $\mathrm{Zn}(\mathrm{OAc})_{2}$ ). The reaction was carried out under the same conditions as in Method A, but instead of $\mathrm{Sc}(\mathrm{OTf})_{3}$ and $\mathrm{N}$-ethyldiisopropylamine, $\mathrm{Zn}(\mathrm{OAc})_{2}$ $(4.6 \mathrm{mg}, 5 \mathrm{~mol} \%)$ as a catalyst was added. The products $\left(R, 1^{\prime} R\right)-17,\left(S, 1^{\prime} R\right)-17,\left(S, 1^{\prime} S\right)-17,\left(R, 1^{\prime} S\right)-17,\left(S, 1^{\prime} S\right)-20$, and $\left(R, 1^{\prime} S\right)-20$ were isolated as in Method A.

4.6.3. Method C (Absence of a catalyst). The reaction of $\mathbf{1 4}$ or $15(1.0 \mathrm{mmol})$ with 1 -phenylethylamine $(154 \mu \mathrm{L}, 1.2 \mathrm{mmol})$ dissolved in toluene $(4 \mathrm{~mL})$ was carried out under argon in a sealed test tube at $80^{\circ} \mathrm{C}$ for 7 days. After the same workup as in Method A (direct chromatography), the products were analyzed by NMR.

(1) $(R, R)-2-(1-P h e n y l e t h y l)$ amino-1-phenyl-ethanol $\left(R, 1^{\prime} R\right)$ 17. White crystals, $41 \mathrm{mg}, 34 \%$ yield, purified by column chromatography $\left(\mathrm{SiO}_{2}, \mathrm{CHCl}_{3}: \mathrm{AcOEt}: \mathrm{MeOH} 1: 1: 0.1\right)$, m.p. $145-146^{\circ} \mathrm{C}$ (lit. [60] m.p. $\left.145-148^{\circ} \mathrm{C}\right),[\alpha]_{D}^{20}=26$ (c 1.0, $\mathrm{CHCl}_{3}$ ), (lit. [60] $[\alpha]_{D}^{20}=26.5\left(c 1.0, \mathrm{CHCl}_{3}\right)$ ), $R_{\mathrm{f}}=0.20$ $\left(\mathrm{CHCl}_{3}: \mathrm{AcOEt}: \mathrm{MeOH} 1: 1: 0.1\right) .{ }^{1} \mathrm{H} \quad \mathrm{NMR}(400 \mathrm{MHz}$, $\left.\mathrm{CDCl}_{3}\right) \delta: 7.34-7.22(\mathrm{~m}, 10 \mathrm{H}), 4.72(\mathrm{dd}, J=8.9,3.7 \mathrm{~Hz}, 1 \mathrm{H})$, $3.75(\mathrm{q}, J=6.4 \mathrm{~Hz}, 1 \mathrm{H}), 2.64(\mathrm{dd}, J=12.2,3.7 \mathrm{~Hz}, 1 \mathrm{H}), 2.54$ (dd, $J=12.2,8.9 \mathrm{~Hz}, 1 \mathrm{H}), 1.38(\mathrm{~d}, J=6.7 \mathrm{~Hz}, 3 \mathrm{H})$. The NMR data are in agreement with literature data for $\left(R, 1^{\prime} R\right)$-enantiomer [60].

(2) (S,R)-2-(1-Phenylethyl)amino-1-phenyl-ethanol $\left(S, 1^{\prime} R\right)$ 17. Brown crystals, $41 \mathrm{mg}, 34 \%$ yield, purified by column chromatography $\left(\mathrm{SiO}_{2}, \mathrm{CHCl}_{3}: \mathrm{AcOEt}: \mathrm{MeOH} 1: 1: 0.1\right)$, $R_{\mathrm{f}}=0.25 \quad\left(\mathrm{CHCl}_{3}:\right.$ AcOEt $\left.: \mathrm{MeOH} \quad 1: 1: 0.1\right) .{ }^{1} \mathrm{H} \quad \mathrm{NMR}$ $\left(400 \mathrm{MHz}, \mathrm{CDCl}_{3}\right) \delta: 7.34-7.22(\mathrm{~m}, 10 \mathrm{H}), 4.55(\mathrm{dd}, J=8.9$, $3.7 \mathrm{~Hz}, 1 \mathrm{H}), 3.81(\mathrm{q}, J=6.4 \mathrm{~Hz}, 1 \mathrm{H}), 2.81(\mathrm{dd}, J=12.2,3.7 \mathrm{~Hz}$, $1 \mathrm{H}), 2.54(\mathrm{dd}, J=12.2,8.9 \mathrm{~Hz}, 1 \mathrm{H}), 1.36(\mathrm{~d}, J=6.7 \mathrm{~Hz}, 3 \mathrm{H})$. The NMR data are in agreement with literature data for $\left(R, 1^{\prime} S\right)$-enantiomer [60].

(3) (S,S)-2-(1-Phenylethyl)amino-1-phenyl-ethanol (S, $\left.1^{\prime} S\right)$ 17. White crystals, $41 \mathrm{mg}, 34 \%$ yield, recrystallized $\left(\mathrm{CH}_{2} \mathrm{Cl}_{2} /\right.$ hexane), $R_{\mathrm{f}}=0.20 \quad\left(\mathrm{CHCl}_{3}:\right.$ AcOEt: $\left.\mathrm{MeOH} 1: 1: 0.1\right) .{ }^{1} \mathrm{H}$ NMR (400 MHz, $\left.\mathrm{CDCl}_{3}\right) \delta: 7.34-7.22(\mathrm{~m}, 10 \mathrm{H}), 4.60$ (dd, $J=8.9,3.7 \mathrm{~Hz}, 1 \mathrm{H}), 3.77(\mathrm{q}, J=6.4 \mathrm{~Hz}, 1 \mathrm{H}), 2.77(\mathrm{dd}, J=12.2$, $3.7 \mathrm{~Hz}, 1 \mathrm{H}), 2.63(\mathrm{dd}, J=12.2,8.9 \mathrm{~Hz}, 1 \mathrm{H}), 1.40(\mathrm{~d}, J=6.7 \mathrm{~Hz}$, $3 \mathrm{H})$. The NMR data are in agreement with literature data for $\left(R, 1^{\prime} R\right)$-enantiomer [60].

(4) (R,S)-2-(1-Phenylethyl)amino-1-phenyl-ethanol $\left(R, 1^{\prime} S\right)$ 17. Brown crystals, $41 \mathrm{mg}, 34 \%$ yield, recrystallized $\left(\mathrm{CH}_{2} \mathrm{Cl}_{2} /\right.$ hexane), m.p. $82-83^{\circ} \mathrm{C}$ (lit. [60] m.p. $80-85^{\circ} \mathrm{C}$ ), $[\alpha]_{D}^{20}=-105$ (c 1.0, $\mathrm{CHCl}_{3}$ ), (lit. [60] $[\alpha]_{D}^{20}=-110$ (c 1.0, $\left.\mathrm{CHCl}_{3}\right)$ ), $R_{\mathrm{f}}=0.25 \quad\left(\mathrm{CHCl}_{3}:\right.$ AcOEt $\left.: \mathrm{MeOH} \quad 1: 1: 0.1\right) .{ }^{1} \mathrm{H} \quad \mathrm{NMR}$ $\left(400 \mathrm{MHz}, \mathrm{CDCl}_{3}\right) \delta: 7.34-7.21(\mathrm{~m}, 10 \mathrm{H}), 4.74(\mathrm{dd}, J=9.2$, $3.4 \mathrm{~Hz}, 1 \mathrm{H}), 3.83(\mathrm{q}, J=6.7 \mathrm{~Hz}, 1 \mathrm{H}), 2.79(\mathrm{dd}, J=12.2,3.7 \mathrm{~Hz}$, $1 \mathrm{H}), 2.55(\mathrm{dd}, J=12.2,9.2 \mathrm{~Hz}, 1 \mathrm{H}), 1.40(\mathrm{~d}, J=6.4 \mathrm{~Hz}, 3 \mathrm{H})$. The NMR data are in agreement with literature data for $\left(R, 1^{\prime} S\right)$-enantiomer [60].

(5) $\left(R / S, 1^{\prime} S\right)-2-\left(1^{\prime}\right.$-Phenylethyl)amino-2-phenyl-ethanol $\left(S, 1^{\prime} S\right)$ 18 and $\left(R, 1^{\prime} S\right)$ - 18, (1:1). Colorless oil, $46 \mathrm{mg}, 38 \%$ total yield, purified by column chromatography $\left(\mathrm{SiO}_{2}, \mathrm{CHCl}_{3}\right.$ : AcOEt : $\mathrm{MeOH} 1: 1: 0.1) .{ }^{1} \mathrm{H}$ NMR $\left(400 \mathrm{MHz}, \mathrm{CDCl}_{3}\right) \delta: 7.40-7,18$ $(\mathrm{m}, 20 \mathrm{H}), 3.90-3.88(\mathrm{~m}, 1 \mathrm{H}), 3.77-3.71(\mathrm{~m}, 2 \mathrm{H}), 3.65-3.67$ $(\mathrm{m}, J=6.7 \mathrm{~Hz}, 1 \mathrm{H}), 3.58-3.50(\mathrm{~m}, 4 \mathrm{H}), 1.36(\mathrm{~d}, J=6.4 \mathrm{~Hz}$, $6 \mathrm{H}) ;{ }^{13} \mathrm{C} \mathrm{NMR}\left(400 \mathrm{MHz}, \mathrm{CDCl}_{3}\right) \delta: 129.1,129.0,128.9$, $128.8,128.7,128.6,128.31,128.30,127.90,127.85,127.8$, 127.7, 127.5, 127.3, 127.2, 126.8, 66.1, 65.9, 62.5, 61.7, 55.5, 55.1, 24.1, 22.2; HR-MS (ESI) $\left[\mathrm{C}_{16} \mathrm{H}_{19} \mathrm{NO}+\mathrm{H}\right]^{+}$requires 242.1539; found 242.1545. The NMR data are in agreement with the reported ones [59].

(6) (1R,2R,1'S)-2-(1'-Phenylethyl)amino-1,2-diphenyl-ethanol $\left(1 R, 2 S, 1^{\prime} S\right)$ - 19. White crystals, $43 \mathrm{mg}, 27 \%$ yield, purified by column chromatography $\left(\mathrm{SiO}_{2}, \mathrm{CHCl}_{3}: \mathrm{AcOEt}: \mathrm{MeOH}\right.$ $1: 1: 0.1$ ), m.p. $135-136^{\circ} \mathrm{C},[\alpha]_{D}^{20}=-63$ (c 0.9, $\mathrm{CHCl}_{3}$ ), (lit. [58] $[\alpha]_{D}^{20}=-66.1$ (c 1.0, $\left.\mathrm{CHCl}_{3}\right) .{ }^{1} \mathrm{H} \mathrm{NMR}(400 \mathrm{MHz}$, $\left.\mathrm{CDCl}_{3}\right) \delta: 7.33-7.13(\mathrm{~m}, 11 \mathrm{H}), 6.98-6.94(\mathrm{~m}, 4 \mathrm{H}), 4.98(\mathrm{~d}$, 
$J=4.9 \mathrm{~Hz}, 1 \mathrm{H}), 4.0(\mathrm{~d}, J=4.9 \mathrm{~Hz}, 1 \mathrm{H}), 3.78(\mathrm{q}, J=6.4 \mathrm{~Hz}$, $1 \mathrm{H}), 1.35(\mathrm{~d}, J=6.7 \mathrm{~Hz}, 3 \mathrm{H}) ;{ }^{13} \mathrm{C}$ NMR $\left(400 \mathrm{MHz}, \mathrm{CDCl}_{3}\right) \delta$ : $145.5,140.5,139.2,128.6,128.2,128.1,127.8,127.5,127.3$, 127.2, 126.6, 126.5, 75.4, 65.6, 54.6, 23.1. The NMR data are in agreement with literature data for $\left(1 R, 2 S, 1^{\prime} S\right)$-enantiomer [58].

(7) (1S,2R, 1'S)-2-(1'-Phenylethyl)amino-1,2-diphenyl-ethanol $\left(1 S, 2 R, 1^{\prime} S\right)$ - 19. Colorless oil, $43 \mathrm{mg}, 27 \%$ yield, purified by column chromatography $\left(\mathrm{SiO}_{2}, \mathrm{CHCl}_{3}\right.$ : $\mathrm{AcOEt}: \mathrm{MeOH}$ $1: 1: 0.1),[\alpha]_{D}^{20}=-111\left(c 1.0, \mathrm{CHCl}_{3}\right)$, (lit. [58] $[\alpha]_{D}^{20}=-$ 112.8 (c 1.0, $\left.\mathrm{CHCl}_{3}\right) .{ }^{1} \mathrm{H}$ NMR $\left(400 \mathrm{MHz}, \mathrm{CDCl}_{3}\right) ; \delta$ : $7.33-7.20(\mathrm{~m}, 10 \mathrm{H}), 7.09-6.95(\mathrm{~m}, 5 \mathrm{H}), 4.64(\mathrm{~d}, J=4.9 \mathrm{~Hz}$, $1 \mathrm{H}), 3.62(\mathrm{~d}, J=4.9 \mathrm{~Hz}, 1 \mathrm{H}), 3.49(\mathrm{q}, J=6.4 \mathrm{~Hz}, 1 \mathrm{H}), 1.23(\mathrm{~d}$, $J=6.7 \mathrm{~Hz}, 3 \mathrm{H}) ;{ }^{13} \mathrm{C} \mathrm{NMR}\left(400 \mathrm{MHz}, \mathrm{CDCl}_{3}\right) \delta: 144.9,140.5$, $139.7,128.5,128.4,128.1,127.8,127.7,127.2,127.1,126.7$, $126.4,75.6,65.8,54.9,24.8$. The NMR data are in agreement with literature data for $\left(1 S, 2 R, 1^{\prime} S\right.$-)-enantiomer [58].

(8) (S,S)-2-(1-Phenylethyl)amino-1-(pyridin-2-yl)ethanol $\left(S, 1^{\prime} S\right)$ - 20. White crystals, $44 \mathrm{mg}, 36 \%$ yield, purified by column chromatography $\left(\mathrm{SiO}_{2}, \mathrm{CHCl}_{3}\right.$ : AcOEt: $\mathrm{MeOH} 1$ : $1: 0.1)$, m.p. $119-120^{\circ} \mathrm{C},[\alpha]_{D}^{20}=-42\left(c 0.6, \mathrm{CH}_{2} \mathrm{Cl}_{2}\right)$. IR $v_{\max }$ (Neat) $3290,2971,1589,1432,703 \mathrm{~cm}^{1} ;{ }^{1} \mathrm{H}$ NMR $(400 \mathrm{MHz}$, $\left.\mathrm{CDCl}_{3}\right) \delta: 8.50-8.48(\mathrm{~m}, 1 \mathrm{H}), 7.64(\mathrm{td}, J=7.4,1.8 \mathrm{~Hz}, 1 \mathrm{H})$, 7.33-7.22 (m, 6H), 7.17-7.14 (m, $1 \mathrm{H}), 4.71(\mathrm{dd}, J=8.1$, $3.7 \mathrm{~Hz}, 1 \mathrm{H}), 3.75(\mathrm{q}, J=6.5 \mathrm{~Hz}, 1 \mathrm{H}), 2.86(\mathrm{dd}, J=12.0,3.7 \mathrm{~Hz}$, $1 \mathrm{H}), 2.69(\mathrm{dd}, J=12.0,8.1 \mathrm{~Hz}, 1 \mathrm{H}), 1.36(\mathrm{~d}, J=6.4 \mathrm{~Hz}, 3 \mathrm{H})$; ${ }^{13} \mathrm{C}$ NMR $\left(400 \mathrm{MHz}, \mathrm{CDCl}_{3}\right) \delta: 161.0,148.5,145.5,136.7$, $128.6,127.1,126.7,122.4,120.6,72.3,58.6,54.4,24.4$; HRMS (ESI) $\left[\mathrm{C}_{15} \mathrm{H}_{18} \mathrm{~N}_{2} \mathrm{O}+\mathrm{H}\right]^{+}$requires 243.1492; found 243.1500 .

(9) (R,S)-2-(1-Phenylethyl)amino-1-(pyridin-2-yl)ethanol $\left(R, 1^{\prime} S\right)$ - 20. White crystals $44 \mathrm{mg}, 36 \%$ yield, purified by column chromatography $\left(\mathrm{SiO}_{2}, \mathrm{CHCl}_{3}: \mathrm{AcOEt}: \mathrm{MeOH} 1\right.$ : $1: 0.1)$, m.p. $75-76^{\circ} \mathrm{C},[\alpha]_{D}^{20}=-52\left(c 0.8, \mathrm{CH}_{2} \mathrm{Cl}_{2}\right)$. IR $v_{\max }$ (Neat) 3081, 2847, 1588, 1433, $702 \mathrm{~cm}^{-1} ;{ }^{1} \mathrm{H}$ NMR $\left(400 \mathrm{MHz}, \mathrm{CDCl}_{3}\right) \delta: 8.48-8.46(\mathrm{~m}, 1 \mathrm{H}), 7.63(\mathrm{td}, J=7.6$, $1.8 \mathrm{~Hz}, 1 \mathrm{H}), 7.37-7.21(\mathrm{~m}, 6 \mathrm{H}), 7.15(\mathrm{~m}, 1 \mathrm{H}) 4.97(\mathrm{dd}, J=8.1$, $3.7 \mathrm{~Hz}, 1 \mathrm{H}), 3.96(\mathrm{q}, J=6.5 \mathrm{~Hz}, 1 \mathrm{H}), 3.08(\mathrm{dd}, J=12.0,3.7 \mathrm{~Hz}$, $1 \mathrm{H}), 2.68(\mathrm{~m}, J=12.0,8.1 \mathrm{~Hz}, 1 \mathrm{H}), 1.50(\mathrm{~d}, J=6.4 \mathrm{~Hz}, 3 \mathrm{H})$; ${ }^{13} \mathrm{C}$ NMR $\left(400 \mathrm{MHz}, \mathrm{CDCl}_{3}\right) \delta: 160.4,148.3,136.9,128.8$, $127.8,127.1,127.0,122.6,120.8,70.9,58.5,53.3,23.3$; HRMS (ESI) $\left[\mathrm{C}_{15} \mathrm{H}_{18} \mathrm{~N}_{2} \mathrm{O}+\mathrm{H}\right]^{+}$requires 243.1492; found 243.1504 .

(10) (1S,2S, 1'S)-2-(1'-Phenylethyl)amino-2-phenyl-1-(pyridin-2-yl)ethanol (1S,2S, $\left.1^{\prime} S\right)$ - 21. White crystals, $42 \mathrm{mg}$, 26\% yield, purified by column chromatography $\left(\mathrm{SiO}_{2}, \mathrm{AcOEt}\right.$ : $\left.\mathrm{CHCl}_{3} 8: 2\right)$, m.p. $94-95^{\circ} \mathrm{C},[\alpha]_{D}^{20}=-78\left(c 1.0, \mathrm{CH}_{2} \mathrm{Cl}_{2}\right)$, $R_{\mathrm{f}}=0.35\left(\mathrm{CHCl}_{3}:\right.$ AcOEt:MeOH $\left.1: 1: 0.1\right)$. IR $v_{\max }$ (Neat) $3147,3032,2924,1592,1433,697 \mathrm{~cm}^{-1}$; ${ }^{1} \mathrm{H}$ NMR $(400 \mathrm{MHz}$, $\left.\mathrm{CDCl}_{3}\right) \delta: 8.36-8.34(\mathrm{~m}, 1 \mathrm{H}), 7.47(\mathrm{td}, J=7.6,1.5 \mathrm{~Hz}, 1 \mathrm{H})$, 7.29-7.24 (m, 4H), 7.24-7.19 (m, 1H), 7.14-7.09 (m, 3H), $7.06-6.94(\mathrm{~m}, 4 \mathrm{H}), 5.12(\mathrm{~d}, J=4.3 \mathrm{~Hz}, 1 \mathrm{H}), 4.15(\mathrm{~d}, J=4.3 \mathrm{~Hz}$, $1 \mathrm{H}), 3.81(\mathrm{q}, J=6.4 \mathrm{~Hz}, 1 \mathrm{H}) 1.35(\mathrm{~d}, J=6.4 \mathrm{~Hz}, 3 \mathrm{H}) ;{ }^{13} \mathrm{C}$ NMR $\left(400 \mathrm{MHz}, \mathrm{CDCl}_{3}\right) \delta: 159.6,148.0,145.77,138.9,136.5$, $128.6,128.2,127.9,127.2,127.1,126.7,122.2,121.5,74.9$,
65.1, 54.8, 23.1; HR-MS (ESI) $\left[\mathrm{C}_{21} \mathrm{H}_{22} \mathrm{~N}_{2} \mathrm{O}+\mathrm{H}\right]^{+}$requires 319.1805; found 319.1801.

(11) (1R,2R, 1'S)-2-(1'-Phenylethyl)amino-2-phenyl-1-(pyridin-2-yl)ethanol(1R,2R, $\left.1^{\prime} S\right)$ - 21. Colorless oil, $42 \mathrm{mg}$, 26\% yield, purified by column chromatography $\left(\mathrm{SiO}_{2}, \mathrm{AcOEt}\right.$ : $\left.\mathrm{CHCl}_{3} 8: 2\right),[\alpha]_{D}^{20}=-93\left(c 1.0, \mathrm{CH}_{2} \mathrm{Cl}_{2}\right), R_{\mathrm{f}}=0.48\left(\mathrm{CHCl}_{3}\right.$ : AcOEt: MeOH $1: 1: 0.1$ ). IR $v_{\max }$ (Neat) 3324, 3026, 2923, $1592,1451,699 \mathrm{~cm}^{-1} ;{ }^{1} \mathrm{H}$ NMR $\left(400 \mathrm{MHz}, \mathrm{CDCl}_{3}\right) \delta: 8.39$ (d, $J=4.6 \mathrm{~Hz}, 1 \mathrm{H}), 7.47(\mathrm{td}, J=7.6,1.5 \mathrm{~Hz}, 1 \mathrm{H}), 7.26-7.12(\mathrm{~m}$, $9 \mathrm{H}), 6.96-6.95(\mathrm{~m}, 3 \mathrm{H}), 4.88(\mathrm{~s}, 1 \mathrm{H}), 4.20(\mathrm{~s}, 1 \mathrm{H}), 3.77(\mathrm{~d}$, $J=4.9 \mathrm{~Hz}, 1 \mathrm{H}), 3.57(\mathrm{q}, J=6.7 \mathrm{~Hz}, 1 \mathrm{H}), 1.30(\mathrm{~d}, J=6.7 \mathrm{~Hz}$, $3 \mathrm{H}) ;{ }^{13} \mathrm{C}$ NMR $\left(400 \mathrm{MHz}, \mathrm{CDCl}_{3}\right) \delta: 159.5,148.0,145.4$, $139.2,136.0,128.4,128.3,128.0,127.2,126.90,126.89,122.4$, 121.8, 76.2, 65.4, 54.9, 25.2; HR-MS (ESI) $\left[\mathrm{C}_{21} \mathrm{H}_{22} \mathrm{~N}_{2} \mathrm{O}+\mathrm{H}\right]^{+}$ requires 319.1805 ; found 319.1796 .

(12) $\left(1 R, 2 R, 1^{\prime} R\right)-2-\left(1^{\prime}-\right.$ Phenylethyl)amino-2-phenyl-1-(pyridin-2-yl)ethanol $\left(1 R, 2 R, 1^{\prime} R\right)-21$. White crystals, $46 \mathrm{mg}, 29 \%$ yield, purified by column chromatography $\left(\mathrm{SiO}_{2}, \mathrm{AcOEt}\right.$ : $\mathrm{CHCl}_{3}$ 8:2), m.p. $94-95^{\circ} \mathrm{C},[\alpha]_{D}^{20}=78$ (c $0.9, \mathrm{CH}_{2} \mathrm{Cl}_{2}$ ), $R_{\mathrm{f}}=0.35\left(\mathrm{CHCl}_{3}:\right.$ AcOEt: MeOH $\left.1: 1: 0.1\right)$. IR $v_{\max }$ (Neat) $3133,3032,2922,1592,1434,697 \mathrm{~cm}^{-1}$; ${ }^{1} \mathrm{H}$ NMR $(400 \mathrm{MHz}$, $\left.\mathrm{CDCl}_{3}\right) \delta: 8.37-8.35(\mathrm{~m}, 1 \mathrm{H}), 7.47(\mathrm{td}, J=7.6,1.5 \mathrm{~Hz}, 1 \mathrm{H})$, 7.32-7.20 (m, 5H), 7.25-7.10 (m, 3H), 7.06-7.03 (m, 1H), 6.99-6.93 (m, 3H), $5.10(\mathrm{~d}, J=4.3 \mathrm{~Hz}, 1 \mathrm{H}), 4.11(\mathrm{~d}, J=4.3 \mathrm{~Hz}$, $1 \mathrm{H}), 3.80(\mathrm{q}, J=6.4 \mathrm{~Hz}, 1 \mathrm{H}) 1.34(\mathrm{~d}, J=6.4 \mathrm{~Hz}, 3 \mathrm{H}) ;{ }^{13} \mathrm{C}$ NMR $\left(400 \mathrm{MHz}, \mathrm{CDCl}_{3}\right) \delta: 159.6,148.1,145.8,139.9,136.0$, 128.6, 128.1, 127.9, 127.2, 127.1, 126.7, 122.2, 121.5, 74.9, 65.1, 54.7, 23.1; HR-MS (ESI) $\left[\mathrm{C}_{21} \mathrm{H}_{22} \mathrm{~N}_{2} \mathrm{O}+\mathrm{H}\right]^{+}$requires 319.1805; found 319.1826 .

(13) (1S,2S, 1'R)-2-(1'-Phenylethyl)amino-2-phenyl-1-(pyridin2-yl)ethanol(1S,2S, $\left.1^{\prime} R\right)$ - 21. Colorless oil, $46 \mathrm{mg}, 29 \%$ yield, purified by column chromatography $\left(\mathrm{SiO}_{2}, \mathrm{AcOEt}: \mathrm{CHCl}_{3}\right.$ $8: 2),[\alpha]_{D}^{20}=93\left(\right.$ c $\left.0.8, \mathrm{CH}_{2} \mathrm{Cl}_{2}\right), R_{\mathrm{f}}=0.48\left(\mathrm{CHCl}_{3}: \mathrm{AcOEt}\right.$ : $\mathrm{MeOH} 1: 1: 0.1$ ). IR $v_{\max }$ (Neat) 3322, 3026, 2923, 1593, $1451,699 \mathrm{~cm}^{-1} ;{ }^{1} \mathrm{H}$ NMR $\left(400 \mathrm{MHz}, \mathrm{CDCl}_{3}\right) \delta: 8.39(\mathrm{~d}$, $J=4.9 \mathrm{~Hz}, 1 \mathrm{H}), 7.49(\mathrm{td}, J=7.2,1.2 \mathrm{~Hz}, 1 \mathrm{H}), 7.28-7.06(\mathrm{~m}$, $9 \mathrm{H}), 6.98-6.90(\mathrm{~m}, 3 \mathrm{H}), 4.88(\mathrm{~d}, J=4.9 \mathrm{~Hz}, 1 \mathrm{H}), 4.22(\mathrm{~s}, 1 \mathrm{H})$, $3.76(\mathrm{~d}, J=4.9 \mathrm{~Hz}, 1 \mathrm{H}), 3.57(\mathrm{q}, J=6.7 \mathrm{~Hz}, 1 \mathrm{H}) 1.30(\mathrm{~d}$, $J=6.7 \mathrm{~Hz}, 3 \mathrm{H}) ;{ }^{13} \mathrm{C}$ NMR $\left(400 \mathrm{MHz}, \mathrm{CDCl}_{3}\right) \delta: 159.5,148.0$, $145.3,139.2,136.0,128.4,128.3,128.0,127.2,126.90,126.89$, $122.4, \quad 121.8, \quad 76.2, \quad 65.4, \quad 54.9, \quad 25.2$; HR-MS (ESI) $\left[\mathrm{C}_{21} \mathrm{H}_{22} \mathrm{~N}_{2} \mathrm{O}+\mathrm{H}\right]^{+}$requires 319.1805; found 319.1811.

(14) $\quad\left(1 R, 2 R, 1^{\prime} S\right)-2-\left(1^{\prime}-C y c l o h e x y l e t h y l\right) a m i n o-2-p h e n y l-1-$ (pyridin-2-yl)ethanol (1R,2R, $\left.1^{\prime} R\right)$ - 22. Brown oil, $53 \mathrm{mg}, 33 \%$ yield, purified by column chromatography $\left(\mathrm{SiO}_{2} \quad 20 \%\right.$ MTBE/hexane), $[\alpha]_{D}^{20}=-45\left(c 0.7, \mathrm{CH}_{2} \mathrm{Cl}_{2}\right)$. IR $v_{\max }$ (Neat) $3139,3019,2924,1435,696 \mathrm{~cm}^{-1}$; ${ }^{1} \mathrm{H}$ NMR $(400 \mathrm{MHz}$, $\left.\mathrm{CDCl}_{3}\right) \delta: 8.46-8.44(\mathrm{~m}, 1 \mathrm{H}), 7.43(\mathrm{dq}, J=7.6,1.8 \mathrm{~Hz}, 1 \mathrm{H})$, $7.14-7.01(\mathrm{~m}, 6 \mathrm{H}), 6.93(\mathrm{~d}, J=7.9 \mathrm{~Hz}, 1 \mathrm{H}), 4.95(\mathrm{~d}, J=4.6 \mathrm{~Hz}$, $1 \mathrm{H}), 4.18(\mathrm{~d}, J=4.6 \mathrm{~Hz}, 1 \mathrm{H}), 2.55-2.52(\mathrm{~m}, 1 \mathrm{H}), 1.72-1.63$ $(\mathrm{m}, 8 \mathrm{H}), 1.28-1.16(\mathrm{~m}, 3 \mathrm{H}), 0.97(\mathrm{~d}, J=6.4 \mathrm{~Hz}, 3 \mathrm{H}) ;{ }^{13} \mathrm{C}$ NMR $\left(400 \mathrm{MHz}, \mathrm{CDCl}_{3}\right) \delta: 160.3,148.2,140.1,136.0,128.0$, $127.8,127.2,122.1,121.5,75.02,65.25,55.0,42.8,29.8,28.1$, 26.9, 26.8, 26.7, 17.8; HR-MS (ESI) $\left[\mathrm{C}_{21} \mathrm{H}_{28} \mathrm{~N}_{2} \mathrm{O}+\mathrm{H}\right]^{+}$requires 325.2274; found 325.2280. 
(15) (1S,2S, 1'R)-2-(1'-Cyclohexylethyl)amino-2-phenyl-1(pyridin-2-yl)ethanol (1S,2S, $1^{\prime} R$ )- 22. Brown oil, $53 \mathrm{mg}, 33 \%$ yield, purified by column chromatography $\left(\mathrm{SiO}_{2} \quad 20 \%\right.$ MTBE/hexane), $[\alpha]_{D}^{20}=28\left(c 0.8, \mathrm{CH}_{2} \mathrm{Cl}_{2}\right)$. IR $v_{\max }$ (Neat) $3062,3028,2925,1434,699 \mathrm{~cm}^{-1}$; ${ }^{1} \mathrm{H}$ NMR $(400 \mathrm{MHz}$, $\left.\mathrm{CDCl}_{3}\right) \delta: 8.42-8.40(\mathrm{~m}, 1 \mathrm{H}), 7.48(\mathrm{dq}, J=7.6,1.8 \mathrm{~Hz}, 1 \mathrm{H})$, $7.15-6.97(\mathrm{~m}, 7 \mathrm{H}), 4.97(\mathrm{~d}, J=4.6 \mathrm{~Hz}, 1 \mathrm{H}), 4.19(\mathrm{~d}, J=4.6 \mathrm{~Hz}$, $1 \mathrm{H}), 2.34-2.31(\mathrm{~m}, 1 \mathrm{H}), 1.73-1.70(\mathrm{~m}, 7 \mathrm{H}), 1.40-0.97(\mathrm{~m}$, $4 \mathrm{H}), 0.92(\mathrm{~d}, J=6.4 \mathrm{~Hz}, 3 \mathrm{H}) ;{ }^{13} \mathrm{C} \mathrm{NMR}\left(400 \mathrm{MHz}, \mathrm{CDCl}_{3}\right) \delta$ : $159.8,148.1,138.9,136.0,128.4,127.8,127.1,122.2,121.6$, 76.2, 65.0, 54.0, 43.9, 29.6, 28.7, 26.8, 26.7, 26.6, 16.7; HR-MS (ESI) $\left[\mathrm{C}_{21} \mathrm{H}_{28} \mathrm{~N}_{2} \mathrm{O}+\mathrm{H}\right]^{+}$requires 325.2274; found 325.2289.

(16) $\quad\left(1 R, 2 R, 1^{\prime} S\right)-2-\left(1^{\prime}\right.$-Phenylethyl)amino-2-phenyl-1-(2,2' bipyridin-6-yl)ethanol $\left(1 R, 2 R, 1^{\prime} S\right)$ - 23. Colorless oil, $60 \mathrm{mg}$, $30 \%$ yield, purified by column chromatography $\left(\mathrm{Al}_{2} \mathrm{O}_{3}, \mathrm{MTBE}\right)$, $R_{\mathrm{f}}=0.48\left(\mathrm{Al}_{2} \mathrm{O}_{3}, \mathrm{MTBE}\right),[\alpha]_{D}^{20}=-112\left(c 0.9, \mathrm{CH}_{2} \mathrm{Cl}_{2}\right) . \mathrm{IR} v_{\max }$ (Neat) 3324, 3026, 2924, 1564, 1430, $699 \mathrm{~cm}^{-11} \mathrm{H}$ NMR $\left(400 \mathrm{MHz}, \mathrm{CDCl}_{3}\right) \delta: 8.64-8.62(\mathrm{~m}, 1 \mathrm{H}), 8.21$ (dd, $J=7.9$, $0.9 \mathrm{~Hz}, 1 \mathrm{H}), 8.05(\mathrm{td}, J=7.9,1.2 \mathrm{~Hz}, 1 \mathrm{H}), 7.74(\mathrm{dd}, J=7.9$, $1.8 \mathrm{~Hz}, 1 \mathrm{H}), 7.65$ (t, $J=7.6 \mathrm{~Hz}, 1 \mathrm{H}), 7.29-7.13(\mathrm{~m}, 9 \mathrm{H}), 7.0-6.98$ (m, 3H), 4.95 (s, $1 \mathrm{H}), 4.22$ (br s, $1 \mathrm{H}), 3.80(\mathrm{~d}, J=4.9 \mathrm{~Hz}, 1 \mathrm{H})$, $3.59(\mathrm{q}, J=6.4 \mathrm{~Hz}, 1 \mathrm{H}), 1.30(\mathrm{~d}, J=6.7 \mathrm{~Hz}, 3 \mathrm{H}) ;{ }^{13} \mathrm{C} \mathrm{NMR}$ $\left(400 \mathrm{MHz}, \mathrm{CDCl}_{3}\right) \delta: 158.8,155.8,154.5,149.2,145.3,139.3$, $137.2,136.8,128.4,128.3,128.0,127.2,126.9,126.8,123.8,121.9$, 121.1, 119.7, 76.2, 65.4, 54.8, 25.2; HR-MS (ESI) $\left[\mathrm{C}_{26} \mathrm{H}_{25} \mathrm{~N}_{3} \mathrm{O}+\mathrm{H}\right]^{+}$requires 396.2070; found 396.2067.

(17) (1S,2S, $\left.1^{\prime} S\right)-2-\left(1^{\prime}\right.$-Phenylethyl)amino-2-phenyl-1-(2,2'bipyridin-6-yl)ethanol (1S,2S,1'S)- 23. Colorless oil, $60 \mathrm{mg}$, $30 \%$ yield, purified by column chromatography $\left(\mathrm{Al}_{2} \mathrm{O}_{3}\right.$, MTBE), $R_{\mathrm{f}}=0.39\left(\mathrm{Al}_{2} \mathrm{O}_{3} \mathrm{MTBE}\right),[\alpha]_{D}^{20}=7.2\left(\mathrm{c} 0.6, \mathrm{CH}_{2} \mathrm{Cl}_{2}\right)$. IR $v_{\max }$ (Neat) 3326, 3025, 2923, 1581, 1564, 1453, 1430, $699 \mathrm{~cm}^{-1}$; ${ }^{1} \mathrm{H}$ NMR $\left(400 \mathrm{MHz}, \mathrm{CDCl}_{3}\right) \delta: 8.62-8.60(\mathrm{~m}, 1 \mathrm{H})$, $8.18(\mathrm{~d}, J=6.7 \mathrm{~Hz}, 1 \mathrm{H}), 7.97(\mathrm{~d}, J=7.9 \mathrm{~Hz}, 1 \mathrm{H}), 7.67(\mathrm{td}$, $J=7.6,1.8 \mathrm{~Hz}, 1 \mathrm{H}), 7.63(\mathrm{t}, J=7.6 \mathrm{~Hz}, 1 \mathrm{H}), 7.31-7.23(\mathrm{~m}$, $6 \mathrm{H}), 7.11-6.94(\mathrm{~m}, 3 \mathrm{H}), 7.01(\mathrm{~d}, J=7.6 \mathrm{~Hz}, 1 \mathrm{H}), 6.96-6.94$ $(\mathrm{m}, 2 \mathrm{H}), 5.14(\mathrm{~d}, J=3.9 \mathrm{~Hz}, 1 \mathrm{H}), 4.21(\mathrm{~d}, J=4.3 \mathrm{~Hz}, 1 \mathrm{H}), 3.82$ $(\mathrm{q}, J=6.4 \mathrm{~Hz}, 1 \mathrm{H}), 1.35(\mathrm{~d}, J=6.4 \mathrm{~Hz}, 3 \mathrm{H}) ;{ }^{13} \mathrm{C} \mathrm{NMR}$ $\left(400 \mathrm{MHz}, \mathrm{CDCl}_{3}\right) \delta: 158.9,155.8,154.5,149.1,145.9,138.9$, $137.2,136.8,129.6,128.1,127.9,127.2,127.1,126.8,123.8$, $121.7,121.1,119.6,74.9,65.2,54.6,22.8$; HR-MS (ESI) $\left[\mathrm{C}_{26} \mathrm{H}_{25} \mathrm{~N}_{3} \mathrm{O}+\mathrm{H}\right]^{+}$requires 396.2070; found 396.2073.

(18) $\left(1 R, 2 R, 1^{\prime} R\right)-2-\left(1^{\prime}\right.$-Cyclohexylethyl)amino-2-phenyl-1-(2,2' bipyridin-6-yl)ethanol $\left(1 R, 2 R, 1^{\prime} R\right)-24$. Colorless oil, $64 \mathrm{mg}$, $32 \%$ yield, purified by column chromatography $\left(\mathrm{Al}_{2} \mathrm{O}_{3}, 20 \%\right.$ MTBE/hexane). $[\alpha]_{D}^{20}=-37\left(c 0.9, \mathrm{CH}_{2} \mathrm{Cl}_{2}\right)$. IR $v_{\max }$ (Neat) $3062,2925,2852,1562,1428,773 \mathrm{~cm}^{-1} ;{ }^{1} \mathrm{H}$ NMR $(400 \mathrm{MHz}$, $\left.\mathrm{CDCl}_{3}\right) \delta: 8.66-8.64(\mathrm{~m}, 1 \mathrm{H}), 8.25(\mathrm{td}, J=7.9,1.2 \mathrm{~Hz}, 1 \mathrm{H})$, $8.19(\mathrm{dd}, J=7.6,0.9 \mathrm{~Hz}, 1 \mathrm{H}) 7.79(\mathrm{td}, J=7.3,1.8 \mathrm{~Hz}, 1 \mathrm{H})$, 7.66-7.58 (m, 1H), 7.31-7.27 (m, 1H), 7.15-6.97 (m, 6H), $5.00(\mathrm{~d}, J=4.6 \mathrm{~Hz}, 1 \mathrm{H}), 4.22(\mathrm{~d}, J=4.6 \mathrm{~Hz}, 1 \mathrm{H}), 2.54-2.51(\mathrm{~m}$, $1 \mathrm{H}), 1.71-1.61(\mathrm{~m}, 6 \mathrm{H}), 1.41-1.32(\mathrm{~m}, 1 \mathrm{H}), 1.25-1.21(\mathrm{~m}$, $1 \mathrm{H}), 1.16-0.97(\mathrm{~m}, 3 \mathrm{H}), 0,95(\mathrm{~d}, J=6.4 \mathrm{~Hz}, 3 \mathrm{H}) ;{ }^{13} \mathrm{C} \mathrm{NMR}$ $\left(400 \mathrm{MHz}, \mathrm{CDCl}_{3}\right) \delta: 159.5,156.1,154.5,149.2,140.3,137.1$, $136.8,128.1,127.9,127.1,123.7,121.7,121.1,119.5,76.3$, 65.6, 65.0, 55.1, 42.7, 29.9, 27.9, 26.8, 26.7, 17.8; HR-MS (ESI) $\left[\mathrm{C}_{26} \mathrm{H}_{31} \mathrm{~N}_{3} \mathrm{O}+\mathrm{H}\right]^{+}$requires 402.2540; found 402.2538.
(19) 2-(1'-Phenylethyl)amino-2-cyclohexyl-1-(1,10-phenanthrolin-2-yl)ethanol $\left(1 R, 2 R, 1^{\prime} R\right)-25$ and $\left(1 S, 2 S, 1^{\prime} R\right)-25$. Colorless oil, $17 \mathrm{mg}, 8 \%$ total yield, purified by column chromatography $\left(\mathrm{SiO}_{2}\right.$, hexane : AcOEt: $\left.\mathrm{CHCl}_{3} 2: 1: 1\right) .{ }^{1} \mathrm{H}$ NMR $\left(400 \mathrm{MHz}, \mathrm{CDCl}_{3}\right) \delta: 9.16-9.14(\mathrm{~m}, 2 \mathrm{H}), 8.22$ (dd, $J=7.9, \quad 1.8 \mathrm{~Hz}, 2 \mathrm{H}), 8.17(\mathrm{~d}, J=8.2 \mathrm{~Hz}, 1 \mathrm{H}), 8.13$ (d, $J=8.2 \mathrm{~Hz}, 1 \mathrm{H}), 7.75(\mathrm{~d}, J=1.8 \mathrm{~Hz}, 4 \mathrm{H}), 7.62-7.58(\mathrm{~m}, 2 \mathrm{H})$, $7.52(\mathrm{~d}, J=8.2 \mathrm{~Hz}, 1 \mathrm{H}), 7.35-7.14(\mathrm{~m}, 11 \mathrm{H}), 3.96(\mathrm{~d}$, $J=3.1 \mathrm{~Hz}, 1 \mathrm{H}), 3.89(\mathrm{dd}, J=7.9,3.4 \mathrm{~Hz}, 1 \mathrm{H}), 3.24(\mathrm{~s}, 1 \mathrm{H})$, $3.22(\mathrm{~d}, J=2.1 \mathrm{~Hz}, 1 \mathrm{H}), 1.79-1.49(\mathrm{~m}, 11 \mathrm{H}), 1.41-1.39(\mathrm{~m}$, 6H), 1.29-1.19 (m, 9H), 1.10-1.09 (m, 4H); HR-MS (ESI) $\left[\mathrm{C}_{28} \mathrm{H}_{31} \mathrm{~N}_{3} \mathrm{O}+\mathrm{H}\right]^{+}$requires 426.2540 ; found 426.2532 .

4.7. General Procedure for the Synthesis of Oxazolidinones from Amino Alcohols. The synthesis of oxazolidinones was performed according to the literature procedure [58]. Triphosgene ( $36 \mathrm{mg}, 0.12 \mathrm{mmol}$ ) was added to a mixture of the amino alcohol $(0.3 \mathrm{mmol})$ in toluene $(3 \mathrm{~mL})$ and potassium carbonate $(57 \mathrm{mg}, 0.41 \mathrm{mmol})$ in water $(1.3 \mathrm{~mL})$ with vigorous stirring at room temperature. After being stirred for $48 \mathrm{~h}$, the mixture was washed with water and brine, the organic layer was dried over $\mathrm{MgSO}_{4}$, filtered, and concentrated in vacuo. The residue was chromatographed on silica gel (hexane/ethyl acetate $7: 3)$ to give the corresponding oxazolidinone.

4.7.1. (4R,5S, $\left.1^{\prime} S\right)-N-\left(1^{\prime}\right.$-Phenylethyl)-4,5-diphenyl-2-oxazolidinone $\left(4 R, 5 S, 1^{\prime} S\right)-26$. White crystals, $60 \mathrm{mg}, 59 \%$ yield, m.p. $154-157^{\circ} \mathrm{C}$ (lit. [58] m.p. $\left.154-156^{\circ} \mathrm{C}\right),[\alpha]_{D}^{20}=19.0$ (c 1.0, $\mathrm{CHCl}_{3}$ ) (lit. [58] $\left.[\alpha]_{D}^{20}=19.1 \quad c \quad 1.0, \mathrm{CHCl}_{3}\right) .{ }^{1} \mathrm{H} \quad \mathrm{NMR}$ $\left(400 \mathrm{MHz}, \mathrm{CDCl}_{3}\right) \delta: 7.39-7.32(\mathrm{~m}, 6 \mathrm{H}), 7.07-7.02(\mathrm{~m}, 6 \mathrm{H})$, 6.95-6.94 (m, 3H), $5.66(\mathrm{~d}, J=8.2 \mathrm{~Hz}, 1 \mathrm{H}), 5.35(\mathrm{q}, J=7.3 \mathrm{~Hz}$, $1 \mathrm{H}), 4.56(\mathrm{~d}, J=8.2 \mathrm{~Hz}, 1 \mathrm{H}), 1.21(\mathrm{~d}, J=7.3 \mathrm{~Hz}, 3 \mathrm{H}) ;{ }^{13} \mathrm{C}$ NMR (400 MHz, $\mathrm{CDCl}_{3}$ ) $\delta: 158.1,140.1,136.6,134.4,128.9$, $128.3,128.2,128.11,128.07,127.9,127.8,127.5,126.0,80.3$, $62.8,53.4,18.3$. The NMR data are in agreement with the literature [58].

4.7.2. (4R,5R, $\left.1^{\prime} S\right)-N-\left(1^{\prime}\right.$-Phenylethyl)-5-pyridin-2-yl-4-phenyl-2-oxazolidinone $\left(4 R, 5 R, 1^{\prime} S\right)-27$. White crystals, $47 \mathrm{mg}$, $46 \%$ yield, m.p. $120-122^{\circ} \mathrm{C},[\alpha]_{D}^{20}=21\left(c 0.8, \mathrm{CHCl}_{3}\right) \cdot{ }^{1} \mathrm{H}$ $\operatorname{NMR}\left(400 \mathrm{MHz}, \mathrm{CDCl}_{3}\right) \delta: 8.24-8.22(\mathrm{~m}, 1 \mathrm{H}), 7.49-7.29(\mathrm{~m}$, $7 \mathrm{H}), 7.13(\mathrm{~d}, J=7.6 \mathrm{~Hz}, 1 \mathrm{H}), 7.05-7.03(\mathrm{~m}, 3 \mathrm{H}), 6.92-6.89$ $(\mathrm{m}, 2 \mathrm{H}), 5.71(\mathrm{~d}, J=7.9 \mathrm{~Hz}, 1 \mathrm{H}), 5.34(\mathrm{q}, J=7.0 \mathrm{~Hz}, 1 \mathrm{H}), 4.79$ $(\mathrm{d}, J=8.2 \mathrm{~Hz}, 1 \mathrm{H}), 1.20(\mathrm{~d}, J=7.34 \mathrm{~Hz}, 3 \mathrm{H}) ;{ }^{13} \mathrm{C} \mathrm{NMR}$ $\left(400 \mathrm{MHz}, \mathrm{CDCl}_{3}\right) \delta: 157.7,154.9,148.7,139.9,136.9,136.3$, $128.9,128.22,128.19,128.1,127.8,127.4,122.4,120.9,80.6$, 61.9, 53.5, 18.2; HR-MS (ESI) $\left[\mathrm{C}_{22} \mathrm{H}_{20} \mathrm{~N}_{2} \mathrm{O}_{2}+\mathrm{H}\right]^{+}$requires 345.1598; found 345.1618 .

4.7.3. (4S,5S, $\left.1^{\prime} S\right)-N-\left(1^{\prime}\right.$-Phenylethyl)-5-pyridin-2-yl-4-phenyl-2oxazolidinone (4S,5S, $\left.1^{\prime} S\right)-27$. White crystals, $47 \mathrm{mg}, 46 \%$ yield, m.p. ${ }^{136}-137^{\circ} \mathrm{C},[\alpha]_{D}^{20}=16\left(c 0.6, \mathrm{CHCl}_{3}\right) .{ }^{1} \mathrm{H} \mathrm{NMR}$ $\left(400 \mathrm{MHz}, \mathrm{CDCl}_{3}\right) \delta: 8.23-8.22(\mathrm{~m}, 1 \mathrm{H}), 7.39(\mathrm{t}, J=7.9 \mathrm{~Hz}$, $1 \mathrm{H}), 7.20-7.12(\mathrm{~m}, 6 \mathrm{H}), 6.93-6.88(\mathrm{~m}, 4 \mathrm{H}), 6.74-6.71(\mathrm{~m}$, $2 \mathrm{H}), 5.81(\mathrm{~d}, J=8.2 \mathrm{~Hz}, 1 \mathrm{H}), 5.07(\mathrm{~d}, J=8.6 \mathrm{~Hz}, 1 \mathrm{H}), 4.62(\mathrm{q}$, $J=7.0 \mathrm{~Hz}, 1 \mathrm{H}), 1.60(\mathrm{~d}, J=7.0 \mathrm{~Hz}, 3 \mathrm{H}) ;{ }^{13} \mathrm{C} \mathrm{NMR}(400 \mathrm{MHz}$, $\left.\mathrm{CDCl}_{3}\right) \delta: 157.4,155.3,148.6,140.4,136.3,134.9,128.4$, $127.93,127.91,127.82,127.80,127.5,122.5,120.9,79.8,64.0$, 
54.6, 18.9; HR-MS (ESI) $\quad\left[\mathrm{C}_{22} \mathrm{H}_{20} \mathrm{~N}_{2} \mathrm{O}_{2}+\mathrm{H}\right]^{+}$requires 345.1598; found 345.1601 .

\section{Data Availability}

All the related data are included in the main text and supplementary files.

\section{Conflicts of Interest}

The authors declare that they have no conflicts of interest.

\section{Acknowledgments}

This work was supported by a subsidy from the Polish Ministry of Science and Higher Education for the Faculty of Chemistry of Wrocław University of Science and Technology.

\section{Supplementary Materials}

This file includes NMR and IR spectra of compounds, a comparison of the ${ }^{1} \mathrm{H}$ NMR and ${ }^{13} \mathrm{C}$ NMR for known and new compounds, DFT computations for ring opening of aryl-heteroaryl-epoxide, and aldol reactions-experimental details and compound characterization. (Supplementary Materials)

\section{References}

[1] J. Zhou, Multicatalyst System in Asymmetric Catalysis, John Wiley \& Sons, Hoboken, NJ, 2015.

[2] L. H. Gade, "Modular assembly of chiral catalysts with polydentate stereodirecting ligands," in Molecular Catalysts: Structure and Functional Design, L. H. Gade and P. Hofmann, Eds., Wiley-VCH, Weinheim, Germany, pp. 313-342, 2014.

[3] Q.-L. Zhou, Privileged Chiral Ligands and Catalysts, WileyVCH, Weinheim, Germany, 2011.

[4] M. Stradiotto and R.-J. Lundgren, Ligand Design in Metal: Chemistry Reactivity and Catalysis, John Wiley \& Sons, Hoboken, NJ, USA, 2016.

[5] A. V. Malkov, I. R. Baxendale, M. Bella et al., "Synthesis of new chiral 2,2'-bipyridyl-type ligands, their coordination to molybdenum(0), copper(II), and palladium(II), and application in asymmetric allylic substitution, allylic oxidation, and cyclopropanation," Organometallics, vol. 20, no. 4, pp. 673690, 2001.

[6] G. Chelucci and R. P. Thummel, "Chiral 2,2'-bipyridines, 1,10-phenanthrolines, and $2,2^{\prime}: 6^{\prime}, 2^{\prime \prime}$-terpyridines: syntheses and applications in asymmetric homogeneous catalysis," Chemical Reviews, vol. 102, no. 9, pp. 3129-3170, 2002.

[7] S. Kobayashi, T. Ogino, H. Shimizu, S. Ishikawa, T. Hamada, and K. Manabe, "Bismuth triflate-chiral bipyridine complexes as water-compatible chiral Lewis acids," Organic Letters, vol. 7, no. 21, pp. 4729-4731, 2005.

[8] A. Bencini and V. Lippolis, "1,10-Phenanthroline: a versatile building block for the construction of ligands for various purposes," Coordination Chemistry Reviews, vol. 254, no. 1718, pp. 2096-2180, 2010.

[9] Y. Nishikawa and H. Yamamoto, "Iron-catalyzed asymmetric epoxidation of $\beta, \beta$-disubstituted enones," Journal of the American Chemical Society, vol. 133, no. 22, pp. 8432-8435, 2011.
[10] M. Liu, S. Ma, Z. Tian et al., "Modular bipyridine ligands coupled with Cinchona alkaloids for $\mathrm{Cu}(\mathrm{II})$-catalyzed asymmetric Henry reactions," Tetrahedron: Asymmetry, vol. 24, no. 12, pp. 736-743, 2013.

[11] L. Zhang, H. Wu, Z. Yang et al., "Synthesis and computation of diastereomeric phenanthroline-quinine ligands and their application in asymmetric Henry reaction," Tetrahedron, vol. 69, no. 49, pp. 10644-10652, 2013.

[12] M. Zielińska-Błajet, P. J. Boratyński, Ł. Sidorowicz, and J. Skarżewski, "Simple approach to modular chiral scaffolds: binding functional sulfur nucleophiles to cinchona alkaloids," Tetrahedron, vol. 72, no. 21, pp. 2643-2648, 2016.

[13] D. S. Belov, E. R. Lukyanenko, A. V. Kurkin, and M. A. Yurovskaya, "Highly stereoselective and scalable synthesis of trans-fused octahydrocyclohepta[b]pyrrol-4(1H)ones via the aza-cope-mannich rearrangement in racemic and enantiopure forms," The Journal of Organic Chemistry, vol. 77, no. 22, pp. 10125-10134, 2012.

[14] U. Iserloh, Y. Wu, J. N. Cumming et al., "Potent pyrrolidineand piperidine-based BACE-1 inhibitors," Bioorganic \& Medicinal Chemistry Letters, vol. 18, no. 1, pp. 414-417, 2008.

[15] S. P. Tanis, J. W. Strohbach, T. T. Parker et al., "The design and development of 2-aryl-2-hydroxy ethylamine substituted $1 \mathrm{H}, 7 \mathrm{H}$-pyrido[1,2,3-de]quinoxaline-6-carboxamides as inhibitors of human cytomegalovirus polymerase," Bioorganic \& Medicinal Chemistry Letters, vol. 20, no. 6, pp. 1994-2000, 2010.

[16] E. N. Jacobsen, "Asymmetric catalysis of epoxide ringopening reactions," Accounts of Chemical Research, vol. 33, no. 6, pp. 421-431, 2000.

[17] A. K. Yudin, Aziridines and Epoxides in Organic Synthesis, Wiley-VCH, Weinheim, Germany, 2006.

[18] Shivani, B. Pujala, and A. K. Chakraborti, "Zinc(II) perchlorate hexahydrate catalyzed opening of epoxide ring by amines: applications to synthesis of (RS)/(R)-Propranolols and (RS)/(R)/(S)-Naftopidils," The Journal of Organic Chemistry, vol. 72, no. 10, pp. 3713-3722, 2007.

[19] C. Bonini and P. Lupattelli, "Synthesis and elaboration of trans 2,3-diaryloxiranes," Arkivoc, vol. 2008, no. 8, pp. 150$182,2008$.

[20] S. Bonollo, D. Lanari, and L. Vaccaro, "Ring-opening of epoxides in water," European Journal of Organic Chemistry, vol. 2011, no. 14, pp. 2587-2598, 2011.

[21] F. A. Saddique, A. F. Zahoor, S. Faiz, S. A. R. Naqvi, M. Usman, and M. Ahmad, "Recent trends in ring opening of epoxides by amines as nucleophiles," Synthetic Communications, vol. 46, no. 10, pp. 831-868, 2016.

[22] M. Lee, J. R. Lamb, M. J. Sanford, A. M. LaPointe, and G. W. Coates, "Nucleophilic ring opening of trans-2,3-disubstituted epoxides to $\beta$-amino alcohols with catalyst-controlled regioselectivity," Chemical Communications, vol. 54, no. 92, pp. 12998-13001, 2018.

[23] R. K. Tak, M. Kumar, M. Nazish, T. K. Menapara, R. I. Kureshy, and N.-U. H. Khan, "Development of recyclable chiral macrocyclic metal complexes for asymmetric aminolysis of epoxides: application for the synthesis of an enantiopure oxazolidine ring," New Journal of Chemistry, vol. 42, no. 18, pp. 15325-15331, 2018.

[24] K. Tanaka, M. Kinoshita, J. Kayahara et al., "Asymmetric ringopening reaction of meso-epoxides with aromatic amines using homochiral metal-organic frameworks as recyclable heterogeneous catalysts," RSC Advances, vol. 8, no. 49, pp. 28139-28146, 2018. 
[25] M. M. Islam, P. Bhanja, M. Halder, S. K. Kundu, A. Bhaumik, and S. M. Islam, "Chiral $\mathrm{Co}$ (iii)-salen complex supported over highly ordered functionalized mesoporous silica for enantioselective aminolysis of racemic epoxides," RSC Advances, vol. 6, no. 111, pp. 109315-109321, 2016.

[26] C. Wang and H. Yamamoto, "Tungsten-catalyzed regio- and enantioselective aminolysis oftrans-2,3-epoxy alcohols: an entry to virtually enantiopure amino alcohols," Angewandte Chemie International Edition, vol. 53, no. 50, pp. 1392013923, 2014.

[27] G. V. More and B. M. Bhanage, "Asymmetric ring opening of meso-epoxides with aromatic amines using $(R)-(+)$-BINOL$\mathrm{Sc}(\mathrm{OTf})_{3}-\mathrm{NMM}$ complex as an efficient catalyst," European Journal of Organic Chemistry, vol. 2013, no. 30, pp. 69006906, 2013.

[28] M. Kumar, R. I. Kureshy, D. Ghosh, N.-U. H. Khan, S. H. R. Abdi, and H. C. Bajaj, "Synthesis of chiral ligands with multiple stereogenic centers and their application in titanium(IV)-catalyzed enantioselective desymmetrization of meso-epoxides," Chem CatChem, vol. 5, no. 8, pp. 2336-2342, 2013.

[29] C. Schneider, A. R. Sreekanth, and E. Mai, "Scandiumbipyridine-catalyzed enantioselective addition of alcohols and amines to meso-epoxides," Angewandte Chemie International Edition, vol. 43, no. 42, pp. 5691-5694, 2004.

[30] E. Mai and C. Schneider, "Scandium-bipyridine-catalyzed enantioselective aminolysis of meso-epoxides," Chemistry-A European Journal, vol. 13, no. 9, pp. 2729-2741, 2007.

[31] S. Azoulay, K. Manabe, and S. Kobayashi, "Catalytic asymmetric ring opening of meso-epoxides with aromatic amines in water," Organic Letters, vol. 7, no. 21, pp. 4593-4595, 2005.

[32] I. Schiffers, T. Rantanen, F. Schmidt, W. Bergmans, L. Zani, and C. Bolm, "Resolution of racemic 2-aminocyclohexanol derivatives and their application as ligands in asymmetric catalysis," The Journal of Organic Chemistry, vol. 71, no. 6, pp. 2320-2331, 2006.

[33] A. T. Placzek, J. L. Donelson, R. Trivedi, R. A. Gibbs, and S. K. De, "Scandium triflate as an efficient and useful catalyst for the synthesis of $\beta$-amino alcohols by regioselective ring opening of epoxides with amines under solvent-free conditions," Tetrahedron Letters, vol. 46, no. 52, pp. 9029-9034, 2005.

[34] A. K. Chakraborti and A. Kondaskar, " $\mathrm{ZrCl}_{4}$ as a new and efficient catalyst for the opening of epoxide rings by amines," Tetrahedron Letters, vol. 44, no. 45, pp. 8315-8319, 2003.

[35] A. K. Chakraborti, S. Rudrawar, and A. Kondaskar, "An efficient synthesis of 2-amino alcohols by silica gel catalysed opening of epoxide rings by amines," Organic \& Biomolecular Chemistry, vol. 2, no. 9, pp. 1277-1280, 2004.

[36] A. K. Chakraborti, S. Rudrawar, and A. Kondaskar, "Lithium bromide, an inexpensive and efficient catalyst for opening of epoxide rings by amines at room temperature under solventfree condition," European Journal of Organic Chemistry, vol. 2004, no. 17, pp. 3597-3600, 2004.

[37] A. K. Chakraborti, A. Kondaskar, and S. Rudrawar, "Scope and limitations of montmorillonite K 10 catalysed opening of epoxide rings by amines," Tetrahedron, vol. 60, no. 41, pp. 9085-9091, 2004.

[38] B. Pujala, S. Rana, and A. K. Chakraborti, "Zinc tetrafluoroborate hydrate as a mild catalyst for epoxide ring opening with amines: scope and limitations of metal tetrafluoroborates and applications in the synthesis of antihypertensive drugs (RS)/(R)/(S)-Metoprolols," The Journal of Organic Chemistry, vol. 76, no. 21, pp. 8768-8780, 2011.
[39] M. Chini, P. Crotti, and F. Macchia, "Metal salts as new catalysts for mild and efficient aminolysis of oxiranes," Tetrahedron Letters, vol. 31, no. 32, pp. 4661-4664, 1990.

[40] M. Chini, P. Crotti, and F. Macchia, "Regioalternating selectivity in the metal salt catalyzed aminolysis of styrene oxide," The Journal of Organic Chemistry, vol. 56, no. 20, pp. 5939-5942, 1991.

[41] M. Sasaki, K. Tanino, A. Hirai, and M. Miyashita, "The C2 selective nucleophilic substitution reactions of 2,3-epoxy alcohols mediated by trialkyl borates: the first endo-mode epoxide-opening reaction through an intramolecular metal chelate," Organic Letters, vol. 5, no. 10, pp. 1789-1791, 2003.

[42] C. Wang and H. Yamamoto, "Nickel-catalyzed regio- and enantioselective aminolysis of 3,4-epoxy alcohols," Journal of the American Chemical Society, vol. 137, no. 13, pp. 43084311, 2015.

[43] C. Wang, L. Luo, and H. Yamamoto, "Metal-catalyzed directed regio- and enantioselective ring-opening of epoxides," Accounts of Chemical Research, vol. 49, no. 2, pp. 193-204, 2016.

[44] F. Azzena, F. Calvani, P. Crotti, C. Gardelli, F. Macchia, and M. Pineschi, "Regiochemical control of the ring opening of 1 : 2 -epoxides by means of chelating processes. 10 . Synthesis and ring opening reactions of mono- and difunctionalized cis and trans aliphatic oxirane systems," Tetrahedron, vol. 51, no. 38, pp. 10601-10626, 1995.

[45] C. Wang and H. Yamamoto, "Tungsten-catalyzed regioselective and stereospecific ring opening of 2,3-epoxy alcohols and 2,3-epoxy sulfonamides," Journal of the American Chemical Society, vol. 136, no. 19, pp. 6888-6891, 2014.

[46] C. Wang and H. Yamamoto, "Tungsten-, molybdenum-, and cerium-promoted regioselective and stereospecific halogenation of 2,3-epoxy alcohols and 2,3-epoxy sulfonamides," Organic Letters, vol. 16, no. 22, pp. 5937-5939, 2014.

[47] V. Bevilacqua, M. King, M. Chaumontet et al., "Copperchelating azides for efficient click conjugation reactions in complex media," Angewandte Chemie International Edition, vol. 53, no. 23, pp. 5872-5876, 2014.

[48] R. A. Poole, G. Bobba, M. J. Cann, J.-C. Frias, D. Parker, and R. D. Peacock, "Synthesis and characterisation of highly emissive and kinetically stable lanthanide complexes suitable for usage "in cellulo"," Organic and Biomolecular Chemistry, vol. 3, no. 6, pp. 1013-1024, 2005.

[49] C. D. Hall and N. Djedovic, "The synthesis and complexation of a cobaltocenium-based redox-active cryptand containing the phenanthroline unit," Journal of Organometallic Chemistry, vol. 648, no. 1-2, pp. 8-13, 2002.

[50] S. Yaragorla, G. Singh, and R. Dada, " $\mathrm{C}_{(\mathrm{sp} 3)^{-H}} \mathrm{H}$ functionalization of methyl azaarenes: a calcium-catalyzed facile synthesis of (E)-2-styryl azaarenes and 2-aryl-1,3-bisazaarenes," Tetrahedron Letters, vol. 56, no. 43, pp. 5924-5929, 2015.

[51] D. Mao, G. Hong, S. Wu, X. Liu, J. Yu, and L. Wang, "Lewisacid-catalyzed benzylic reactions of 2-methylazaarenes with aldehydes," European Journal of Organic Chemistry, vol. 2014, no. 14, pp. 3009-3019, 2014.

[52] A. Solladie'-Cavallo, P. Lupattelli, C. Marsol et al., "Opening of diaryl epoxides: ortho-fluorophenyl and 2-pyridyl epoxides," European Journal of Organic Chemistry, vol. 2002, no. 8, pp. 1439-1444, 2002.

[53] P. Saisaha, C. Nerungsi, S. Iamsaard, and T. Thongpanchang, "Pyridine stabilized oxiranyl remote anions," Tetrahedron Letters, vol. 50, no. 29, pp. 4217-4220, 2009.

[54] A. Solladié-Cavallo, M. Roje, T. Isarno, V. Sunjic, and V. Vinkovic, "Pyridyl and furyl epoxides of more than $99 \%$ 
enantiomeric purities: the use of a phosphazene base," European Journal of Organic Chemistry, vol. 2000, no. 6, pp. 1077-1080, 2000.

[55] G. He, S.-Y. Zhang, W. A. Nack, R. Pearson, J. Rabb-Lynch, and G. Chen, "Total synthesis of hibispeptin a via Pd-catalyzed $\mathrm{C}\left(\mathrm{sp}^{3}\right)-\mathrm{H}$ arylation with sterically hindered aryl iodides," Organic Letters, vol. 16, no. 24, pp. 6488-6491, 2014.

[56] P. Wu, G. Santoni, M. Fröba, and D. Rehder, "Modelling the sulfoxygenation activity of vanadate-dependent peroxidases," Chemistry \& Biodiversity, vol. 5, no. 10, pp. 1913-1926, 2008.

[57] A. Reyes and E. Juaristi, "Convenient route for the preparation of C2-symmetric (+)-(2R,3R)- and (-)- $(2 S, 3 S)-2,3-$ diphenylaziridine," Chirality, vol. 10, no. 1-2, pp. 95-99, 1998.

[58] S. Sugiyama, K. Morishita, M. Chiba, and K. Ishii, "Chemoselective debezylation of the N-1-phenylethyl goup in 2oxazolidinones by the anisole-methanesulfonic acid system," Heterocycles, vol. 57, pp. 637-648, 2002.

[59] K. Higashiyama, H. Inoue, T. Yamauchi, and H. Takahashi, "Asymmetric synthesis of $\left(1 R, 1^{\prime} R\right)$ - and $\left(1 S, 1^{\prime} S\right)$-bis(1-arylethyl)amines by way of a diastereoselective addition to chiral imines and oxaxolidines with organometallic reagents," Journal of the Chemical Society, Perkin Transactions 1, no. 2, pp. 111-115, 1995.

[60] A. Iuliano, D. Pini, and P. Salvadori, "Optically active N-1phenylethyl derivatives of (1R)-2-amino-1-phenylethanol as chiral auxiliaries in the enantioselective addition of diethylzinc to arylaldehydes," Tetrahedron: Asymmetry, vol. 6, no. 3, pp. 739-744, 1995.

[61] S. Gamsey, K. DeLaTorre, and B. Singaram, "Asymmetric hydrogenation of chiral vinyloxazaborolidines under ambient conditions," Tetrahedron: Asymmetry, vol. 16, no. 3, pp. 711-715, 2005.

[62] E. Wojaczyńska, J. Skarżewski, Ł. Sidorowicz, R. Wieczorek, and J. Wojaczyński, "Zinc complexes formed by 2,2'-bipyridine and 1,10-phenanthroline moieties combined with 2azanorbornane: modular chiral catalysts for aldol reactions," New Journal of Chemistry, vol. 40, no. 11, pp. 9795-9805, 2016. 

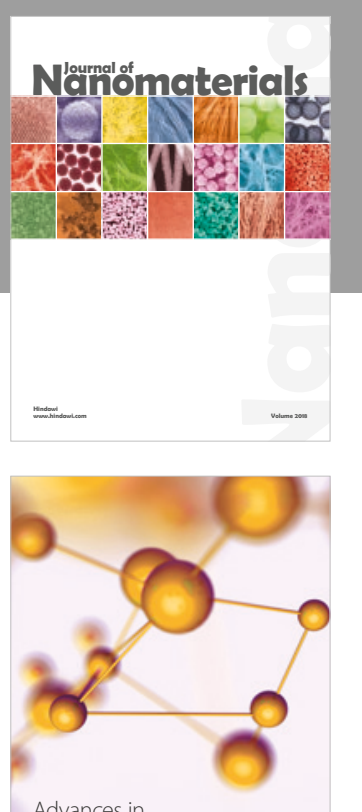

Physical Chemistry
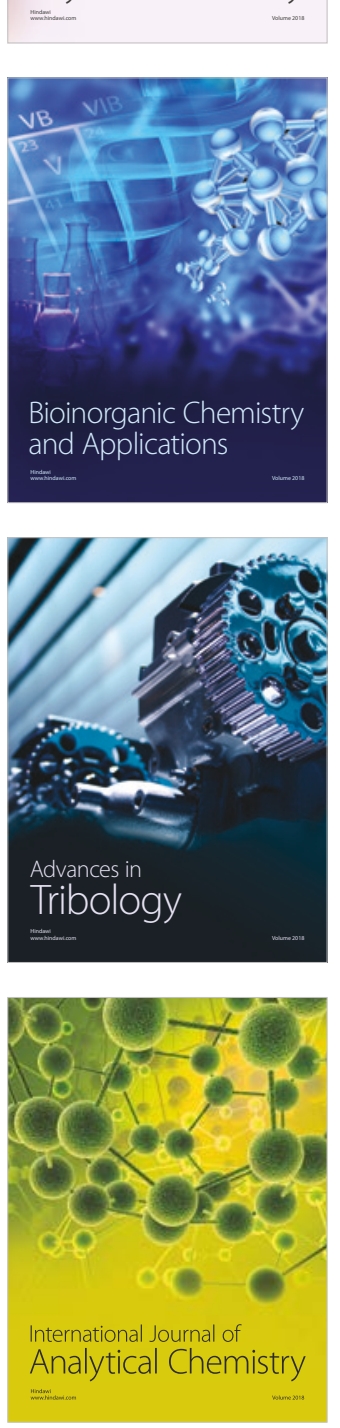

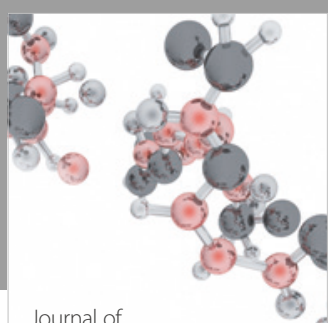

Analytical Methods

in Chemistry

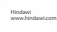

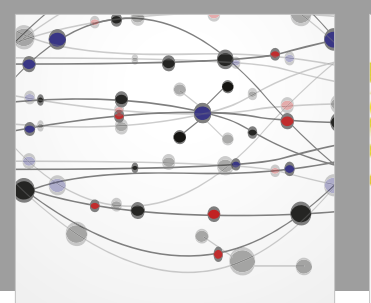

The Scientific World Journal

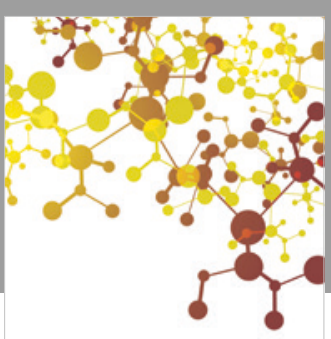

Journal of

Applied Chemistry
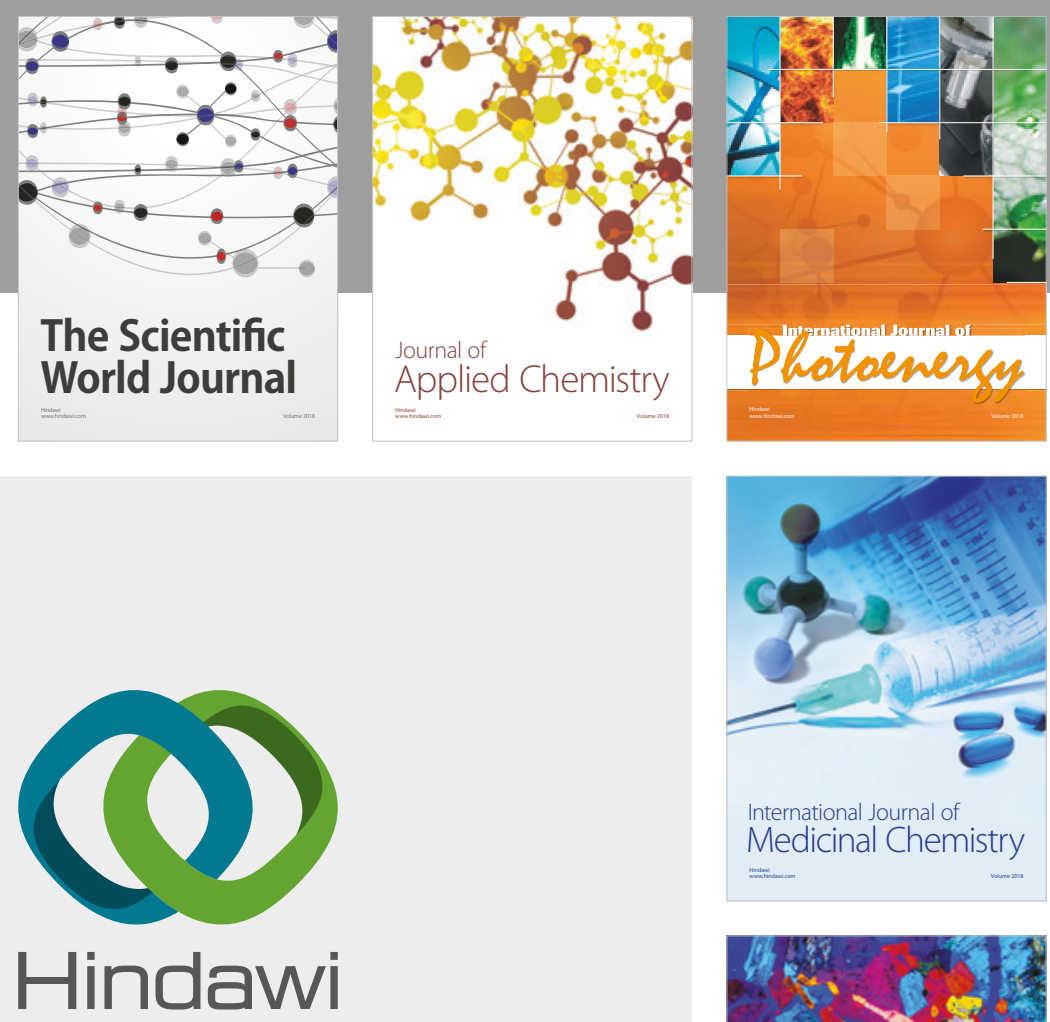

Submit your manuscripts at

www.hindawi.com
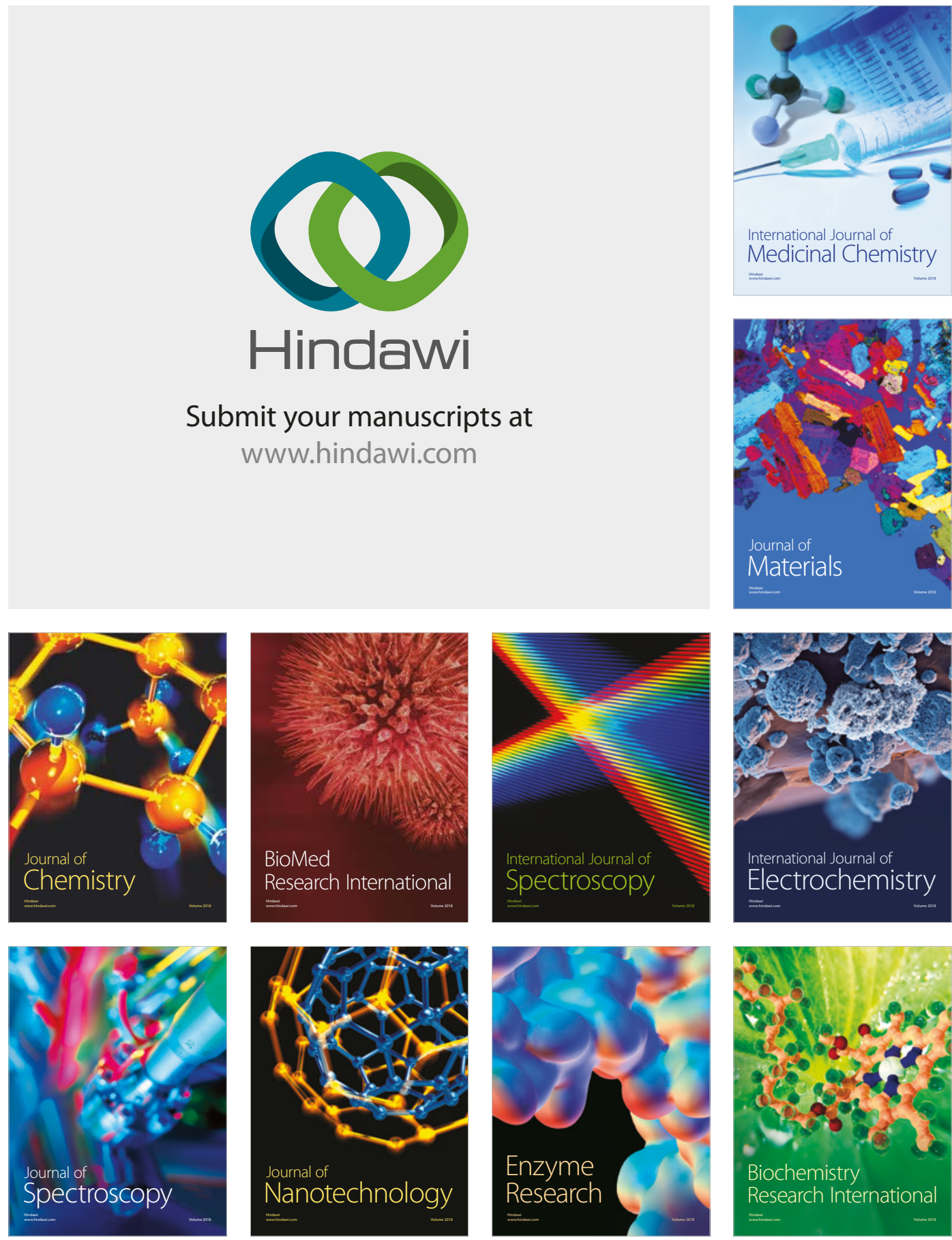
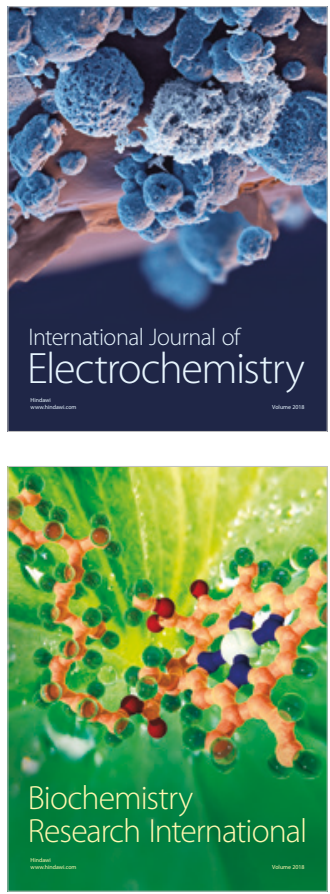